OPEN ACCESS

Edited by:

Xihui Shen,

Northwest A\&F University, China

Reviewed by:

Yufeng Yao,

Shanghai Jiao Tong University, China

Christian U. Riedel,

Universität UIm, Germany

${ }^{*}$ Correspondence:

Birgitte H. Kallipolitis bhk@bmb.sdu.dk

Specialty section: This article was submitted to Infectious Diseases,

a section of the journal Frontiers in Microbiology

Received: 10 January 2018 Accepted: 15 March 2018

Published: 27 March 2018

Citation: dos Santos PT, Menendez-Gil P, Sabharwal D, Christensen J-H, Brunhede MZ, Lillebæk EMS and

Kallipolitis BH (2018) The Small Regulatory RNAs LhrC1-5 Contribute to the Response of Listeria monocytogenes to Heme Toxicity. Front. Microbiol. 9:599. doi: 10.3389/fmicb.2018.00599

\section{The Small Regulatory RNAs LhrC1-5 Contribute to the Response of Listeria monocytogenes to Heme Toxicity}

\author{
Patrícia T. dos Santos, Pilar Menendez-Gil, Dharmesh Sabharwal, \\ Jens-Henrik Christensen, Maja Z. Brunhede, Eva M. S. Lillebæk and \\ Birgitte H. Kallipolitis*
}

Department of Biochemistry and Molecular Biology, University of Southern Denmark, Odense, Denmark

The LhrC family of small regulatory RNAs (sRNAs) is known to be induced when the foodborne pathogen Listeria monocytogenes is exposed to infection-relevant conditions, such as human blood. Here we demonstrate that excess heme, the core component of hemoglobin in blood, leads to a strong induction of the LhrC family members LhrC1-5. The heme-dependent activation of IhrC1-5 relies on the response regulator LisR, which is known to play a role in virulence and stress tolerance. Importantly, our studies revealed that LhrC1-5 and LisR contribute to the adaptation of L. monocytogenes to excess heme. Regarding the regulatory function of the sRNAs, we demonstrate that LhrC1-5 act to down-regulate the expression of known LhrC target genes under heme-rich conditions: oppA, tcs $A$, and lapB, encoding surface exposed proteins with virulence functions. These genes were originally identified as targets for LhrC-mediated control under cell envelope stress conditions, suggesting a link between the response to heme toxicity and cell envelope stress in L. monocytogenes. We also investigated the role of $\mathrm{LhrC1-5}$ in controlling the expression of genes involved in heme uptake and utilization: Imo2186 and Imo2185, encoding the heme-binding proteins Hbp1 and Hbp2, respectively, and Imo0484, encoding a heme oxygenase-like protein. Using in vitro binding assays, we demonstrated that the LhrC family member LhrC4 interacts with mRNAs encoded from Imo2186, Imo2185, and Imo0484. For Imo0484, we furthermore show that LhrC4 uses a CU-rich loop for basepairing to the AG-rich Shine-Dalgarno region of the mRNA. The presence of a link between the response to heme toxicity and cell envelope stress was further underlined by the observation that LhrC1-5 down-regulate the expression of Imo0484 in response to the cell wallacting antibiotic cefuroxime. Collectively, this study suggests a role for the LisR-regulated sRNAs LhrC1-5 in a coordinated response to excess heme and cell envelope stress in L. monocytogenes.

Keywords: Listeria monocytogenes, heme toxicity, cell envelope stress, sRNA, two-component system, target mRNA 


\section{INTRODUCTION}

Listeria monocytogenes is a Gram-positive, foodborne pathogen and the causative agent of listeriosis (Vazquez-Boland et al., 2001). To successfully establish an infection, this facultative intracellular pathogen must overcome several obstacles, such as low iron availability in the host (Chipperfield and Ratledge, 2000; Stojiljkovic and Perkins-Balding, 2002; Lungu et al., 2009). Upon infection, bacterial pathogens face a significant challenge in accessing iron, as it is mostly complexed to iron-binding proteins, such as hemoglobin, ferritin, lactoferrin, and transferrin (Hentze et al., 2004; Hammer and Skaar, 2011; Huang and Wilks, 2017). Bacterial pathogens are known to overcome this phenomenon, called nutritional immunity (Kochan, 1973; Wakeman and Skaar, 2012), through the activation of diverse mechanisms that allow iron acquisition from the iron-binding proteins in the host. Two-thirds of the total iron in the human body are sequestered in erythrocytes as heme bound to hemoglobin (Cassat and Skaar, 2013). In order to use heme as a source for iron, the pathogens first need to lyse erythrocytes, bind hemoglobin or other host heme proteins, and then extract and import the heme molecule for intracellular degradation to liberate free iron (Choby and Skaar, 2016). The iron acquisition system in L. monocytogenes has been the focus of diverse studies that have identified several specific iron transport and storage proteins (McLaughlin et al., 2011; Lechowicz and Krawczyk-Balska, 2015), such as Fhu, involved in the uptake of ferrichrome siderophores, and HupDGC, involved in the uptake of hemoglobin and hemin (i.e., the oxidized version of heme) (Jin et al., 2006; Xiao et al., 2011). Notably, HupDGC in L. monocytogenes is homologous to the well-studied iron-regulated surface determinant system (Isd) from Staphylococcus aureus (Skaar and Schneewind, 2004; Reniere et al., 2007). When the heme concentration in the environment is below $50 \mathrm{nM}$, heme acquisition in L. monocytogenes occurs with the participation of the heme-binding proteins 1 and 2, Hbp1 and Hbp2 (encoded by $l m o 2186$ and $l m o 2185$, respectively), which are anchored in the cell wall by Sortase B (Xiao et al., 2011). While a role for $\mathrm{Hbp} 1$ is still unclear, Hbp2 is known to scavenge for heme and hemoglobin and facilitate the transport of heme through the cell wall (Xiao et al., 2011; Klebba et al., 2012; Malmirchegini et al., 2014). Heme can then cross the membrane through the HupDGC ABC transporter (Jin et al., 2006; Xiao et al., 2011). At higher heme concentrations, free heme molecules are predicted to diffuse through the porous structure of peptidoglycan. Then, they are bound by HupD anchored to the cytoplasmic membrane and transported into the cell (Klebba et al., 2012; Lechowicz and Krawczyk-Balska, 2015). Once inside the cell, heme may be used, for example, as a cofactor for several enzymes, such as catalases and peroxidases, as a respiratory cofactor for oxygen transport and storage, or as a catalyst of electron transfer (Chiabrando et al., 2014). Alternatively, heme can be broken down by heme oxygenases, like the characterized Isd-type heme-degradation enzyme Isd-LmHde and/or the IsdG homolog Lmo0484, to liberate free iron (Wu et al., 2005; TravagliniAllocatelli, 2013; Duong et al., 2014). To maintain intracellular iron homeostasis, L. monocytogenes possesses an iron-binding protein, Fur (ferric uptake regulator), which negatively regulates several genes under iron-replete conditions, including the genes encoding Hbp1 and Hbp2 (Ledala et al., 2010). Fur boxes have also been identified upstream from other genes coding for proteins involved in the heme uptake and utilization system, such as HupDGC and IsdG/Lmo0484 (McLaughlin et al., 2012), suggesting that a tight regulatory connection between the iron and heme uptake/utilization systems is crucial for L. monocytogenes. Conversely, while heme can be an essential source of iron for L. monocytogenes upon infection, during certain pathological states, severe hemolysis may occur, resulting in high levels of free heme (up to $20 \mu \mathrm{M}$ ) (Arruda et al., 2004). As heme is a highly reactive lipophilic molecule, the cells must protect themselves against the potential damaging effects of heme under heme-rich conditions, such as in the bloodstream and blood-rich organs (McLaughlin et al., 2011; Choby and Skaar, 2016; Huang and Wilks, 2017). For L. monocytogenes, the mechanism by which this pathogen senses and responds to excess heme is yet to be characterized.

The LhrC family in L. monocytogenes consists of seven sibling sRNAs with regulatory roles under infection-relevant conditions (Thorsing et al., 2017). The family includes the highly homologous sRNAs LhrC1-5, as well as Rli22 and Rli33-1, which share lower homology. $r$ li22 and $l h r C 1-5$ are positively regulated by the two-component system (TCS) LisRK that responds to cell envelope stress, whereas the general stress sigma factor $\sigma^{\mathrm{B}}$ controls the expression of rli33-1 (Sievers et al., 2014; Mollerup et al., 2016). The seven siblings are induced under various stress conditions and act to modulate the expression of specific target genes by an antisense mechanism. So far, three genes have been shown to be negatively regulated by the LhrCs at the post-transcriptional level: $\operatorname{lap} B$, encoding a cell wall anchored virulence adhesin; $o p p A$, encoding a substrate-binding protein of an oligopeptide transporter; and tcs $A$, encoding a CD4 ${ }^{+}$T cell-stimulating antigen (Sievers et al., 2014, 2015). For the mRNA targets characterized so far, the LhrC sRNAs act by basepairing to the Shine-Dalgarno (SD) region, leading to inhibition of translation initiation and/or decreased mRNA levels (Sievers et al., 2014, 2015; Mollerup et al., 2016). Strikingly, all seven sRNAs are highly induced when L. monocytogenes is exposed to human blood, suggesting an important regulatory role for the LhrC family in this host environment (Toledo-Arana et al., 2009). Yet, the specific component(s) in blood leading to induction of the LhrCs are presently unknown.

In this study, we aimed to investigate if the induction of the LhrC sRNAs by human blood could be linked to the increasing levels of heme in the bloodstream upon infection. Indeed, L. monocytogenes has the ability to secrete listeriolysin $\mathrm{O}$ (LLO) that facilitates the release of hemoglobin through erythrocyte lysis (Parrisius et al., 1986; Geoffroy et al., 1987; Foller et al., 2007). Similar to the LhrC family, hly, the gene encoding LLO, is also highly expressed in human blood, which supports the hypothesis that L. monocytogenes possibly encounters increasing levels of heme after host invasion (ToledoArana et al., 2009). Here, we demonstrate the LisR-dependent induction of LhrC family members, in particular LhrC1-5, 
by hemin, and show their capacity to regulate their known target genes under hemin stress conditions. In addition, we provide evidence that LhrC1-5 and LisR contribute to the adaptation of L. monocytogenes to excess hemin. Finally, we propose a role for LhrC1-5 in the post-transcriptional control of the heme uptake and utilization genes lmo0484, Imo2185, and $l m o 2186$ in response to cell envelope stress and excess hemin.

\section{MATERIALS AND METHODS}

\section{Bacterial Strains and Growth Conditions}

The wild-type strain used in this study was L. monocytogenes serotype $1 / 2$ c strain LO28 (Vazquez-Boland et al., 1992). The isogenic mutant derivatives LO28 $\Delta / h r C 1-5$ and LO28 $\Delta$ lisR were constructed in previous work (Kallipolitis et al., 2003; Sievers et al., 2014). The remaining isogenic mutant derivatives of this strain were constructed as previously described (Christiansen et al., 2004) by using the temperature-sensitive shuttle vector pAUL-A (Schaferkordt and Chakraborty, 1995). Primers used for in-frame deletions are listed in Supplementary Table S1. All strains used in this study are listed in Supplementary Table S2. L. monocytogenes was routinely grown at $37^{\circ} \mathrm{C}$ with aeration in brain heart infusion broth (BHI, Oxoid) unless stated otherwise. When appropriate, cultures were supplemented with kanamycin $(50 \mu \mathrm{g} / \mathrm{mL})$ or erythromycin $(5 \mu \mathrm{g} / \mathrm{mL})$. For induction of sRNA expression, cultures were supplemented with cefuroxime ( $4 \mu \mathrm{g} / \mathrm{mL}$, corresponding to $9 \mu \mathrm{M}$ ) or various concentrations of hemin (Sigma). Hemin is the commercially available version of heme, which contains the oxidized $\mathrm{Fe}^{3+}$ ferric form instead of the reduced $\mathrm{Fe}^{2+}$ ferrous form. For experimental purposes, we will refer to hemin, while for the discussion we will refer to heme instead. Hemin was dissolved in $1.4 \mathrm{M} \mathrm{NaOH}$ and stock solutions were prepared fresh every time. In stress tolerance assays, overnight cultures were diluted to $\mathrm{OD}_{600}=0.002$ into $\mathrm{BHI}$ adjusted with various concentrations of hemin, or into BHI, when hemin was added to exponentially growing cells $\left(\mathrm{OD}_{600}=0.2\right)$; growth was monitored until strains reached stationary phase. For cloning of plasmid vectors, Escherichia coli TOP10 cells (Invitrogen) were grown with aeration in Luria-Bertani broth supplemented with kanamycin $(50 \mu \mathrm{g} / \mathrm{mL})$ or erythromycin $(150 \mu \mathrm{g} / \mathrm{mL})$, when appropriate.

\section{Plasmid Constructions and $\beta$-Galactosidase Assays}

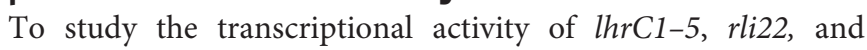
rli33-1, we used the promoter-less lacZ transcriptional fusion vector pTCV-lac (Poyart and Trieu-Cuot, 1997) fused to the promoter regions of the seven sRNA-encoding genes constructed previously (Sievers et al., 2014; Mollerup et al., 2016). Post-transcriptional regulation of LhrC target genes was monitored using in-frame translational lacZ fusions of $l a p B$ constructed in previous work (Sievers et al., 2014), and of 1 mo0484 constructed in the present study. Briefly, DNA fragments encoding a moderate promoter (Sievers et al., 2014) as well as a region spanning the SD region of lmo0484 ( -48 to +47 , relative to translation start site) were ligated into pCK-lac (Nielsen et al., 2010). For the $\beta$-galactosidase assay, L. monocytogenes strains carrying the plasmids were grown overnight, diluted to $\mathrm{OD}_{600}=0.02$ into fresh $\mathrm{BHI}$ and grown to $\mathrm{OD}_{600}=0.2$ (for strains with translational fusions) or $\mathrm{OD}_{600}=0.35$ (for strains with transcriptional fusions). Cultures were then split and stressed with either $9 \mu \mathrm{M}$ cefuroxime or $8 \mu \mathrm{M}$ hemin for 1 and/or $2 \mathrm{~h}$. Samples $(1 \mathrm{~mL})$ were withdrawn prior to stress and at the indicated time points after the subjected stress. $\beta$-galactosidase assays were conducted as previously described (Christiansen et al., 2004). As the applied stress conditions resulted in impaired growth relative to the non-stress control condition, a direct comparison between the stressed and non-stressed cultures was not possible. However, the growth of the wild-type and mutant strains were comparable under each of the conditions tested (i.e., control, cefuroxime or hemin stress, respectively). Therefore, the $\beta$-galactosidase activities of wild-type and mutant strains were analyzed for each of the conditions using two-tailed Student's $t$-test (i.e., wild-type, stressed vs. mutant, stressed). Only differences with at least $95 \%$ confidence were reported as statistically significant.

\section{RNA Isolation and Purification}

For primer extension and northern blot analysis, L. monocytogenes was grown to $\mathrm{OD}_{600}=0.35$. Cultures where then split, stressed with the indicated stressor concentration and samples were taken $(10 \mathrm{~mL})$ at the indicated time points. Cells were harvested by centrifugation at $11,000 \mathrm{rcf}$ for $3 \mathrm{~min}$ at $4^{\circ} \mathrm{C}$, and snap-frozen in liquid nitrogen. Cells were disrupted by the FastPrep instrument (Bio101, Thermo Scientific Corporation) and total RNA was extracted using TRI Reagent (Molecular Research Center, Inc.) as previously described (Nielsen et al., 2010). The integrity, concentration and purity of the RNA were confirmed by agarose gel electrophoresis, and NanoDrop 2000 or DeNovix DS-11 Fx+.

\section{Primer Extension}

Primer extension experiments were performed as previously described (Christiansen et al., 2004). The ${ }^{32}$ P-labeled, single-stranded primers used for detection of $1 m o 0484$ and lmo2186 transcription start sites are listed in Supplementary Table S1.

\section{Northern Blotting}

Total RNA $(10 \mu \mathrm{g})$ was resolved on a $6 \%$ or $8 \%$ polyacrylamide8 M urea gel as previously described (Nielsen et al., 2010); alternatively, $20 \mu \mathrm{g}$ of total RNA was separated on a formaldehyde agarose gel for $3 \mathrm{~h}$ and $15 \mathrm{~min}$ prior to capillarity blotting on a Zeta-Probe membrane (Bio-Rad) (Sheehan et al., 1995). Membranes were hybridized with ${ }^{32}$ P-labeled DNA singlestranded probes listed in Supplementary Table S1. RNA bands were visualized using a Typhoon Trio or a Typhoon FLA9000 (GE Healthcare) and analyzed with IQTL 8.0 quantification software (GE Healthcare). 


\section{Reverse Transcriptase-Quantitative Polymerase Chain Reaction (RT-qPCR)}

Fifty $\mu \mathrm{g}$ of total RNA was DNase-treated according to the manufacturer (Roche), and further purified with phenol-chloroform extraction. Three $\mu \mathrm{g}$ of DNA-free RNA was used for cDNA synthesis using the Maxima First Strand cDNA Synthesis Kit (Fermentas), following the manufacturer's recommendations. RT-qPCR was performed using SYBR Green PCR Master Mix (Fermentas) and specific primer sets for the gene of interest (Supplementary Table S1). The samples were run on a MX3000 quantitative PCR thermocycler (Stratagene) with an initial step at $95^{\circ} \mathrm{C}$ for $10 \mathrm{~min}, 40$ cycles of $15 \mathrm{~s}$ at $95^{\circ} \mathrm{C}$, $30 \mathrm{~s}$ at $60^{\circ} \mathrm{C}$ and $30 \mathrm{~s}$ at $72^{\circ} \mathrm{C}$. Data was analyzed using the Relative Expression Software Tool - Multiple Condition Solver REST-MCS version 2 (Pfaffl, 2001; Pfaffl et al., 2002). The tpi and $r p o B$ genes served as reference genes. The experiment was carried out in three biological replicates, each in technical duplicates. Statistical differences were analyzed with a randomization test provided in the REST software. Only differences with at least $95 \%$ confidence were reported as statistically significant.

\section{In Silico Predictions}

The IntaRNA software (Busch et al., 2008; Wright et al., 2014; Mann et al., 2017) was used for predicting interactions between target mRNAs and sRNAs. Full length sequences of sRNAs and truncated versions of the targets were employed. Secondary structure predictions were obtained through Mfold (Zuker, 2003).

\section{Electrophoretic Mobility Shift Assays (EMSAs)}

Templates for in vitro transcription carried a T7 RNA polymerase binding site at their $5^{\prime}$-end and were generated by PCR. Templates for $l m o 0484$ were obtained by PCR from chromosomal DNA and sRNA transcripts were made using overlapping primers (Supplementary Table S1). In vitro transcription, RNA purification, de-phosphorylation and labeling were performed as described previously (Sievers et al., 2014). EMSAs were performed as previously described (Nielsen et al., 2010). Briefly, $40 \mathrm{fmol}$ of $5^{\prime}$-end labeled RNA was incubated with excess unlabeled RNA in a total volume of $10 \mu \mathrm{L}$ in the presence of non-specific competitor (tRNA) for $1 \mathrm{~h}$ at $37^{\circ} \mathrm{C}$ followed by 10 min on ice. Samples were separated on a $5 \%$ non-denaturing gel at $4^{\circ} \mathrm{C}$. RNA bands were visualized and analyzed as described for the northern blotting experiments.

\section{In Vitro Enzymatic Structure Probing}

$5^{\prime}$-end labeled transcripts were prepared as described for the EMSAs. The enzymatic probing was carried out as previously described (Sievers et al., 2014), with some deviations. Briefly, for the alkaline hydrolysis ladder, $0.2 \mathrm{pmol}$ of labeled RNA was mixed with alkaline hydrolysis buffer (Ambion) and $10 \mu \mathrm{g}$ of yeast tRNA (Ambion) in a total volume of $10 \mu \mathrm{L}$ and incubated at $95^{\circ} \mathrm{C}$ for $5 \mathrm{~min}$; for $\mathrm{T} 1$ control sample, $0.2 \mathrm{pmol}$ of labeled RNA was denatured and incubated with $0.01 \mathrm{U}$ of T1 RNase (Ambion) for $5 \mathrm{~min}$. Structure probing RNA interactions were incubated at $37^{\circ} \mathrm{C}$ for $1 \mathrm{~h}$ before treating the samples with the indicated cleaving agent: $0.01 \mathrm{U}$ T1 RNase for $5 \mathrm{~min}$ and 0.0015 U V1 RNase (Ambion) for $2 \mathrm{~min}$. Control samples were prepared likewise (except for the cleaving agents) and incubated at $37^{\circ} \mathrm{C}$ for the duration of the experiment. Samples were placed on ice and mixed with $2 \times$ loading buffer type II (Ambion). Five $\mu \mathrm{L}$ of each sample was separated on an $8 \%$ denaturing polyacrylamide gel. RNA bands were visualized and analyzed as described for the northern blotting experiments.

\section{RESULTS}

\section{LhrC1-5 Are Highly Induced by Hemin in a LisR-Dependent Manner}

To investigate if LhrC1-5 are induced in response to hemin exposure, the sRNA levels were determined via northern blot analysis on total RNA purified from L. monocytogenes LO28 wild-type cells subjected to various concentrations of hemin for $1 \mathrm{~h}$. As a control, wild-type cells were exposed to a sub-inhibitory concentration of the cell wall-acting antibiotic cefuroxime $(9 \mu \mathrm{M})$, which is already known as an inducer of LhrC1-5 (Sievers et al., 2014). As seen in Figure 1A, LhrC1-5 levels were strongly induced with increasing concentrations of hemin $(0,1,2,4,8$, and $16 \mu \mathrm{M})$ and this induction was not caused by the hemin dissolvent $\mathrm{NaOH}(\mathrm{C} 1$ and $\mathrm{C} 2)$. In addition, exposure to $8 \mu \mathrm{M}$ hemin induced $\mathrm{LhrC1}-5$ to the same extent as $9 \mu \mathrm{M}$ cefuroxime (Cef). The novel members of the $\mathrm{LhrC}$ family, Rli22 and Rli33-1, were also investigated through the same means to assess their induction under hemin stress conditions (Supplementary Figure S1A). Rli22 appeared to be induced when L. monocytogenes was exposed to the highest concentrations of hemin $(8$ and $16 \mu \mathrm{M})$, whereas the expression of Rli33-1 remained constant under all conditions tested.

To further investigate the induction of $\mathrm{LhrC1}-5$ by hemin, the promoter activity of the five $\operatorname{lhr} C$ copies was determined using transcriptional fusions of each promoter to the reporter gene lacZ in the vector pTCV-lac (Sievers et al., 2014). As the TCS LisRK has been shown to play a role in the activation of $l h r C 1-5$ under cell envelope stress conditions (Sievers et al., 2014), both the wild-type strain and a mutant strain lacking the response regulator LisR $(\Delta$ lisR) were transformed with the promoter-lac $Z$ plasmids. The $\beta$-galactosidase activity was determined $2 \mathrm{~h}$ after subjecting the cultures to hemin stress $(8 \mu \mathrm{M})$ and non-stressed cultures were included as controls (Figure 1B). In line with our previous observations, the promoter-lac $Z$ constructs gave rise to $\beta$-galactosidase activity close to background levels under non-stress conditions (Sievers et al., 2014). However, after $2 \mathrm{~h}$ of hemin stress, a significant increase in the $\beta$-galactosidase activity was observed in the wild-type strain carrying the $l h r C$-lacZ fusion plasmids, while no increase in activity was detected in the $\Delta$ lis $R$ cells. The activity of rli22 and rli33-1 promoters fused to lacZ was also determined in wild-type cells (Supplementary Figure S1B). Notably, the $\beta$-galactosidase activity in non-stressed and stressed cultures remained similar, showing that these promoters were not significantly activated by the addition of hemin. Overall, the results confirmed the induction of $\mathrm{LhrC} 1-5$ by hemin exposure 


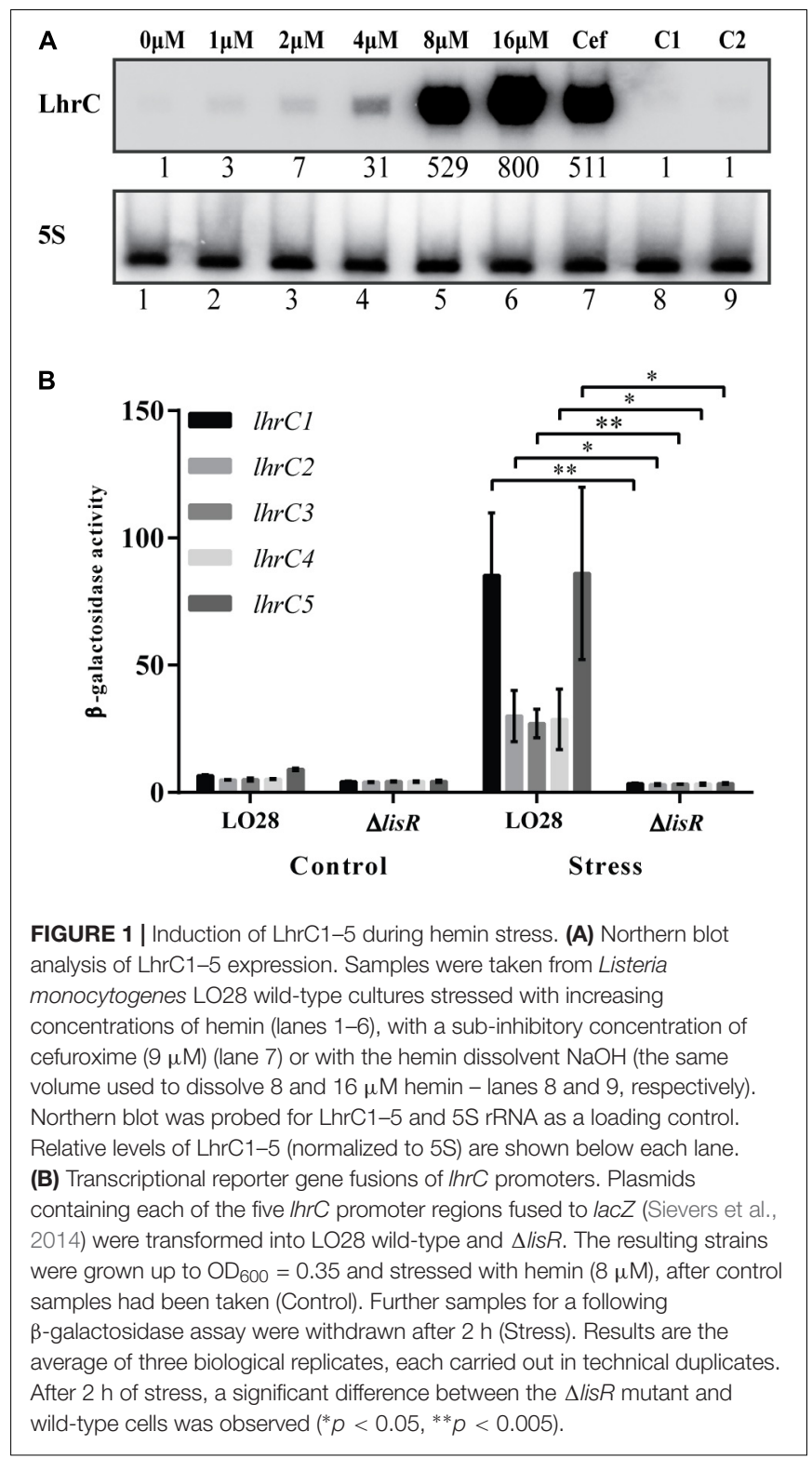

and the requirement of LisR in the regulation of $1 \mathrm{hrCl}-5$ when L. monocytogenes faces excess concentrations of hemin. In contrast, the effect of hemin on rli22 and rli33-1 appeared to be negligible, and for that reason the following experiments will only focus on $\mathrm{LhrC1}-5$.

\section{LhrC1-5 and LisR Play a Role in the Adaptation to Hemin Toxicity}

To investigate whether LhrC1-5 and/or the response regulator LisR contribute to the adaptation of $L$. monocytogenes to hemin stress, growth of the wild-type strain and strains lacking LhrC1-5 or LisR was compared when these cultures were exposed to $16 \mu \mathrm{M}$ hemin. Hemin was either added to the cultures at the beginning of the growth experiment (Figure 2A), or when the cells reached the early exponential phase (Figure 2B). No difference in growth was observed between the wild-type and the two mutant strains when hemin was added at time $0 \mathrm{~h}$ (Figure 2A). However, when hemin was added to exponentially growing cells, the strains clearly responded differently. As seen in Figure 2B, both mutant strains struggled to adapt to the stress condition compared to the wild-type, suggesting the involvement of LhrC1-5 and LisR in the adaptation of L. monocytogenes to excess hemin.

\section{LhrC1-5 Down-Regulate Their Known Target Genes Under Hemin Stress}

Three genes (lapB, oppA, and $t c s A$ ) were previously identified as direct targets of LhrC1-5 (Sievers et al., 2014, 2015). More specifically, LhrC1-5 down-regulate their expression at the post-transcriptional level in response to cefuroxime stress. This raises the question whether $\mathrm{LhrC1}-5$ regulate the same set of targets, no matter the stress factor causing their induction, or if LhrC1-5 have different targets under different stress conditions. To investigate if $\mathrm{LhrC1}-5$ control the expression of $o p p A$ and $t c s A$ under hemin stress, we made use of northern blot analysis to assess the oppA and tcs $A$ mRNA levels in wild-type and $\Delta l h r C 1-5$ strains. The cultures were grown to early exponential phase and subjected to $8 \mu \mathrm{M}$ hemin for $1 \mathrm{~h}$; non-stressed cultures were included as controls (Figure 3A). In wild-type cells, hemin exposure resulted in a minor decrease in $о p p A$ mRNA (less than 1.5-fold), whereas for the $l h r C 1-5$ mutant strain, the oppA mRNA level was 4-fold higher, when comparing hemin stressed and non-stressed cells. For tcs $A$, a threefold decrease was seen when wild-type cells were subjected to hemin stress, whereas no major changes were observed when comparing the levels of $t c s A$ mRNA in stressed and non-stressed cultures of the $\Delta l h r C 1-5$ strain. Altogether, the northern blot analysis demonstrated that the induction of $\mathrm{LhrC} 1-5$ by hemin diminishes the expression of $o p p A$ and causes down-regulation of $t$ cs $A$ (Figure 3A). Strikingly, the regulatory effect observed for LhrC1-5 on oppA and tcs $A$ is comparable to the one obtained when using cefuroxime as an inducer of LhrC1-5 (Sievers et al., 2015). Clearly, LhrC1-5 down-regulate the mRNA levels of oppA and $\operatorname{tcs} A$ to a similar extent in response to both cefuroxime and hemin stresses.

As $l a p B$ is part of a large operon, a reporter gene fusion strategy was used to assess the effect of hemin-induced LhrC sRNAs on this target gene (Sievers et al., 2014). In the pC-lapB-lacZ construct, a sequence including the $5^{\prime}$ untranslated region and the first codons of lapB's coding region was fused downstream of a moderate promoter and inserted in-frame to lacZ in vector pCK-lac (Sievers et al., 2014). Notably, the moderate promoter was not affected by LhrC1-5 under hemin stress (Supplementary Figure S2A). The $\beta$-galactosidase activities were measured at time 0 and $2 \mathrm{~h}$ relative to the onset of hemin stress $(8 \mu \mathrm{M})$, and nonstressed cultures were included as controls (Figure $3 \mathbf{B}$ and Supplementary Figure S2B). After hemin exposure, the $\Delta l h r C 1-5$ cells containing $\mathrm{pC}$-lapB-lac $Z$ showed higher $\beta$-galactosidase activity relative to the wild-type cells, whereas no difference in activity was observed under non-stress (control) conditions. These results demonstrate that $\mathrm{LhrC1}-5$ down-regulate this 
A

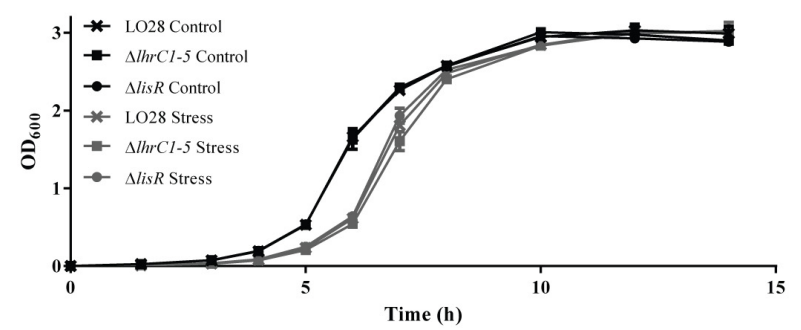

B

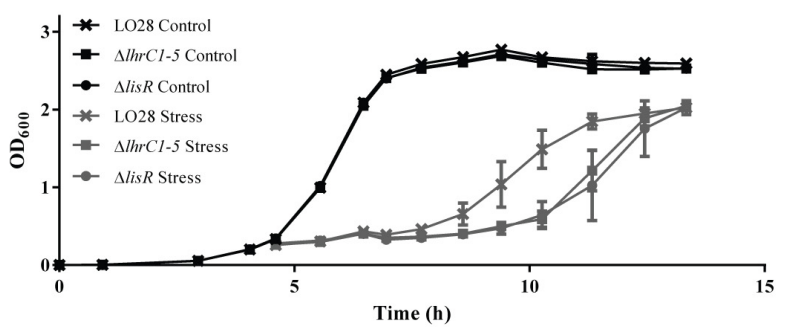

FIGURE 2 | Stress tolerance assay. (A) LO28 wild-type, $\Delta / h r C 1-5$ and $\Delta$ lis $R$ strains were grown in BHI (Control) and BHI containing $16 \mu \mathrm{M}$ hemin (Stress). (B) LO28 wild-type, $\Delta / h r C 1-5$ and $\Delta$ lisR were grown in $\mathrm{BHI}$ to $\mathrm{OD}_{600}=0.2$. Then, the cultures were split in two; one half was stressed with $16 \mu \mathrm{M}$ hemin (Stress) and the other half kept unstressed (Control). Bacterial growth was monitored until all cultures reached stationary phase. For each assay, the average of three biological replicates is shown.

target gene at the post-transcriptional level in response to hemin stress.

\section{Proteins Related to Heme Uptake and Utilization Are Affected by LhrC1-5 Under Cefuroxime Stress}

In a previous study, Sievers et al. (2015) performed transcriptomic and proteomic analyses of LO28 wild-type and $\Delta l h r C 1-5$ cells to identify genes controlled by LhrC1-5. For the transcriptome study, the cultures were subjected to cefuroxime stress for $30 \mathrm{~min}$ to induce $\mathrm{LhrC1}-5$ regulation, whereas $1 \mathrm{~h}$ of cefuroxime stress was chosen for the proteomic analysis. These studies generated two lists of genes that were significantly up- or down-regulated in the lhrC1-5 mutant strain relative to the wild-type at the RNA and protein levels, respectively. By then, tight parameters were employed to select the genes to be further characterized as potential targets of LhrC1-5. Thus, only genes that were regulated at least 1.5 -fold by LhrC1-5 at the RNA level and 2.0-fold at the protein level in all three biological replicates were chosen for further investigation (Sievers et al., 2015). Only three genes passed these strict criteria, including $o p p A$ and $t c s A$. Finding that LhrC1-5 also act as regulatory sRNAs in response to hemin stress prompted us to search these lists for potential targets related to heme uptake and utilization. Indeed, when searching through the data obtained from the proteomic analysis, we found that Lmo0484 was more than threefold up-regulated in $\Delta l h r C 1-5$ relative to the wild-type strain in all three biological replicates (Sievers et al., 2015). Lmo0484 is a homolog of the IsdG heme oxygenase from $S$. aureus, which degrades exogenous heme in the cytoplasm, leading to the release of free iron to be used as a nutrient source (Wu et al., 2005). In addition, we found that in two of the three biological replicates, the proteins Lmo2186 and Lmo2185 were more than three and twofold up-regulated, respectively, in the $\Delta l h r C 1-5$ strain relative to wild-type (Sievers et al., 2015). Lmo2186 and Lmo2185 are homologs of IsdC from S. aureus (Newton et al., 2005) and were previously characterized as heme-binding proteins 1 and 2 ( $\mathrm{Hbp} 1$ and $\mathrm{Hbp} 2$ ), respectively (Xiao et al., 2011). Even though none of these genes were significantly affected by LhrC1-5 at the RNA level following
$30 \mathrm{~min}$ of cefuroxime stress (Sievers et al., 2015), the results obtained from the proteomic analysis made it relevant to further investigate the regulatory effect of LhrC1-5 on Imo0484, lmo2186, and lmo2185.

In regard to the known mode of action of $\mathrm{LhrC1}-5$, we hypothesized that the sRNAs might act on $l m o 0484, \operatorname{lmo} 2186$, and lmo2185 via direct binding to the mRNAs, leading to inhibition of translation initiation. To investigate this assumption, we first performed in silico analyses of the potential basepairing between the sRNAs and mRNAs. Using IntaRNA (Busch et al., 2008; Wright et al., 2014; Mann et al., 2017), we predicted that LhrC1-5 could bind to the SD region of $l m o 0484, l m o 2185$, and $l m o 2186$ mRNAs (Figures 4A-C and Supplementary Figure S3). To verify experimentally the binding of the LhrCs to these mRNAs, EMSAs were performed, where $\mathrm{LhrC} 4$ was used as a representative of the LhrC family (Figures 4D-F). 5' -end labeled LhrC4 was visibly able to bind all three RNAs, where a single shifted band appeared with increasing concentrations of unlabeled lmo0484, Imo2185, or Imo2186 RNA. These results clearly demonstrate that LhrC4 interacts with these mRNAs (Figure 4).

\section{LhrC4 Loop A Binds to the SD Region of Imo0484 mRNA}

Based on the results obtained from the proteomic analysis and the in vitro binding studies, we decided to further analyze the basepairing between the sRNA LhrC4 and Imo0484 mRNA. All five LhrCs hold three single-stranded CU-rich regions known to interact with their target mRNAs (Sievers et al., 2014): loop A, a single-stranded stretch and the terminator loop. To investigate the importance of the CU-rich regions for the interaction with 1 mo0484 mRNA, mutant versions of LhrC4, where the entire CU-rich region is mutated, were tested for their ability to bind wild-type $1 m 00484$ in an EMSA [for details about the mutations, see Sievers et al. (2014)]. The LhrC4 mutant derivatives were labeled and mixed with increasing concentrations of $\operatorname{lmo} 0484$ RNA (Figure 5A). The results revealed that mutations in the single-stranded stretch (LhrC4_mut_2) and in the terminator loop (LhrC4_mut_3) did not reduce the interaction between the two RNAs, whereas the loop A mutation (LhrC4_mut_4) abolished the basepairing, 


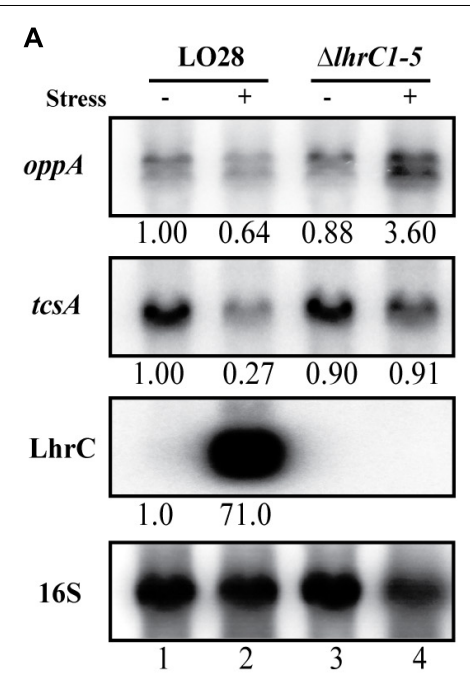

B

B $\quad$ C-lapB-lacZ

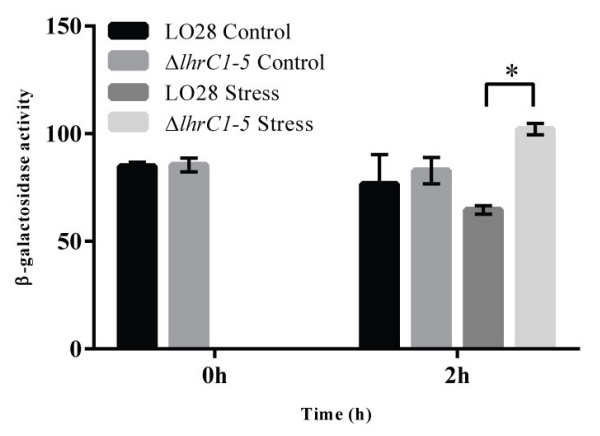

FIGURE 3 | LhrC-mediated down-regulation of the known target genes oppA, tcs $A$ and lapB. (A) Northern blot analysis of oppA mRNA, tcsA mRNA and LhrC1-5. Samples were taken from LO28 wild-type and $\Delta / h r C 1-5$ cultures exposed to $8 \mu \mathrm{M}$ hemin stress for $1 \mathrm{~h}(+)$ as well as from non-stressed cultures (-). Northern blots were probed for oppA mRNA, tcsA mRNA, LhrC1-5 and 16S rRNA (loading control). Relative levels of oppA mRNA, tcsA mRNA and LhrC1-5 (normalized to 16S) are shown below each lane. (B) $\beta$-galactosidase assay of LO28 wild-type and $\Delta / h r C 1-5$ strains carrying a translational reporter gene fusion of lapB to lac $Z$ in the vector pCK-lac (Sievers et al., 2014). $\beta$-galactosidase activities of wild-type and mutant cells were measured at the indicated time points under non-stress conditions (Control) and after exposure to $8 \mu \mathrm{M}$ hemin (Stress). The results are the average of three biological replicates, each carried out in technical duplicates. After $2 \mathrm{~h}$ of stress, a significant difference (asterisk) between the mutant and wild-type cells was observed $(p<0.0001)$.

suggesting that this region is crucial for the binding of LhrC4 to lmo0484 (Figure 5A). To investigate if the SD region of $l m o 0484$ is responsible for basepairing to $\mathrm{LhrC} 4$ loop A, Imo0484 was mutated in two of the seven nucleotides in the predicted $\mathrm{LhrC} 4$ binding region (Figure 5B). Interestingly, the mutated version of lmo0484 (lmo0484_MUT) was not able to bind the wild-type version of LhrC4 (Figure 5C). Finally, we created a mutant version of LhrC4 containing the complementary nucleotide mutations of lmo0484_MUT in loop A (LhrC4_loopA_MUT) (Figure 5B). As expected, the LhrC4_loopA_MUT variant was unable to bind the wild-type version of 1 mo0484, but when testing the binding of LhrC4_loopA_MUT to lmo0484_MUT, the interaction was restored (Figure 5C), confirming the importance of loop A for basepairing to the SD region of $1 m o 0484$ mRNA.

To study the structural implications of the interaction between the two RNAs, structural probing was employed. First, $5^{\prime}$-end labeled $\mathrm{LhrC} 4$ was subjected to RNase hydrolysis in the absence and presence of unlabeled lmo0484 RNA (Figure 6A). The enzymes RNase T1 and RNase V1 were used to cleave the RNAs, where the former is specific for unpaired guanine and the latter for double-stranded regions. The region from nucleotide 37 to 43, which resides in loop A, presented an increased V1 cleavage when $\mathrm{LhrC} 4$ interacts with 1 mo0484 (Figures 6A,C). Thus, the nucleotides residing in loop A went from single-stranded to double-stranded upon binding to lmo0484, confirming the importance of loop A for the sRNA-mRNA interaction. The V1 protection of nucleotides 51-56 and the increased T1 cleavage of G48 and G52 suggest that binding of loop A to lmo0484 disrupts the double-stranded structure of stem A. An increased V1 cleavage was also seen at nucleotides $67-69$, corresponding to the single-stranded stretch in LhrC4, indicating that this CU-rich region may play a role in the basepairing of $\mathrm{LhrC} 4$ to $l m o 0484$ as well, but to a lesser extent than loop A. Similarly, the region from nucleotides 79-82, residing in the double-stranded region of the terminator loop, obtained a more single-stranded conformation (V1 protection) in the presence of $1 m o 0484$, suggesting an opening of the terminator stem upon basepairing between the target mRNA and the single-stranded stretch (Figures 6A,C). To corroborate these results, $5^{\prime}$-end labeled lmo0484 RNA was subjected to the action of the same RNases in the absence and presence of unlabeled $\mathrm{LhrC} 4$ (Figure 6B). The nucleotides from -8 to -13 , corresponding to the putative $\mathrm{SD}$ region of lmo0484, changed from single-stranded to double-stranded conformation (from -8 to -10 : increased cleaving by V1; from -11 to -13 : protection from T1) (Figures 6B,D). This result clearly supports that the SD region is bound by LhrC4. Additionally, some nucleotides shifted from double-stranded to single-stranded conformations ( -27 to $-29,-15$ to -18 and +8 to +10 ), most likely reflecting changes in the secondary structure of lmo0484 occurring as a consequence of LhrC4 basepairing to the SD region (Figures 6B,D). Collectively, the results of the EMSAs and structure binding experiments support that the CUrich region of loop A, and to a lesser extent the single-stranded stretch, binds to the SD region of $1 m o 0484$ mRNA.

\section{LhrC1-5 Control the Expression of Imo0484 at the Post-transcriptional Level in Response to Cefuroxime Stress}

After showing that LhrC4 basepairs to the SD region of lmo0484 mRNA in vitro, we aimed to test the mechanism by which LhrC1-5 regulate this target in vivo. To this end, we analyzed the regulatory effect of LhrC1-5 at the post-transcriptional level on $l m o 0484$ by making use once again of the lac $Z$ reporter strategy. First, the $5^{\prime}$-end of the $1 m 00484$ transcript was mapped by primer extension analysis to position -48 relative to the translation start site (Supplementary Figure S4). Then, a sequence encoding the $5^{\prime}$-end of the $l m o 0484$ transcript 


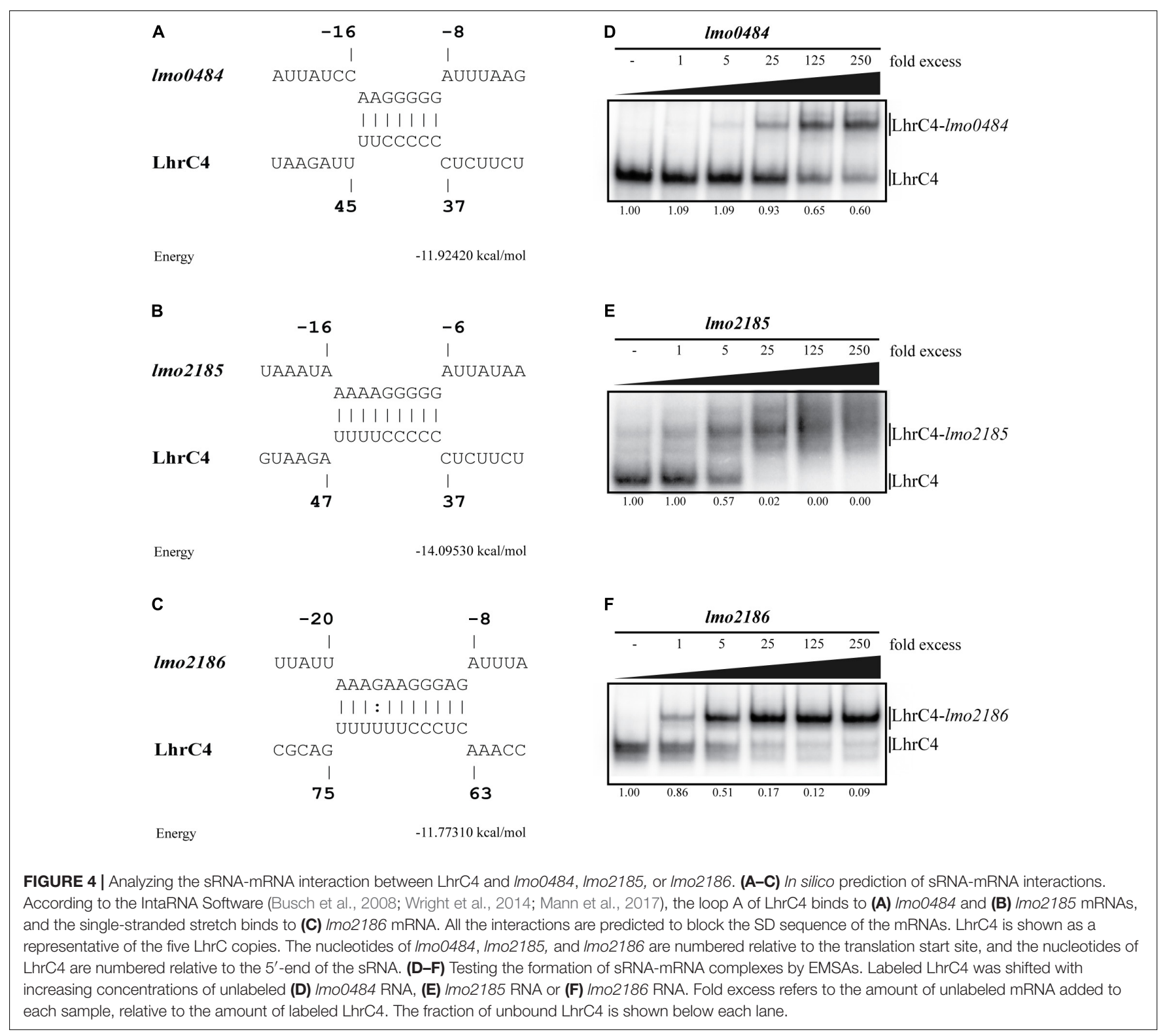

and additional $47 \mathrm{bp}$ of 1 mo0484 coding region was fused downstream of a moderate promoter and fused in-frame to lac $Z$ in the vector pCK-lac (Nielsen et al., 2010). The generated $\mathrm{pC}$-lmo0484-lacZ construct was transformed into both wild-type and $\Delta l h r C 1-5$ cells. The resulting strains were then subjected to cefuroxime stress for 1 or $2 \mathrm{~h}$, and the $\beta$-galactosidase activity was measured. During cefuroxime stress, higher activity levels were obtained in $\Delta / h r C 1-5$ cells compared to wild-type cells, confirming that LhrC1-5 down-regulate the expression of $1 m o 0484$ at the post-transcriptional level in response to cefuroxime exposure (Figure 7A and Supplementary Figure S2C).

As 1 mo0484 was not significantly affected by LhrC1-5 at the RNA level after 30 min of cefuroxime stress (Sievers et al., 2015), we performed RT-qPCR on total RNA purified from cells exposed to cefuroxime for $1 \mathrm{~h}$, where the level of $\mathrm{LhrC} 1-5$ induction is known to be at its highest (Sievers et al., 2014); non-stressed samples were included as controls. The lmo0484 mRNA level was quantified in $\Delta l h r C 1-5$ cells relative to wild-type cells under non-stress (control) and stress conditions (Figure 7B). In the control samples, the mRNA ratio was approximately 1 , showing that there was no difference in 1 mo0484 expression in the wild-type and mutant strains grown under non-stress conditions. In contrast, lmo0484 mRNA expression was 1.75-fold higher in $\Delta l h r C 1-5$ compared to wild-type after $1 \mathrm{~h}$ of cefuroxime stress. Collectively, the results of the $\beta$-galactosidase assay and the RT-qPCR experiment demonstrate that LhrC1-5 down-regulate $\operatorname{lmo0484}$ expression at the post-transcriptional level upon subjecting $L$. monocytogenes to cefuroxime stress. Thus, Imo0484 is clearly a target for LhrC1-5-mediated control when L. monocytogenes is exposed to cell envelope stress conditions. 


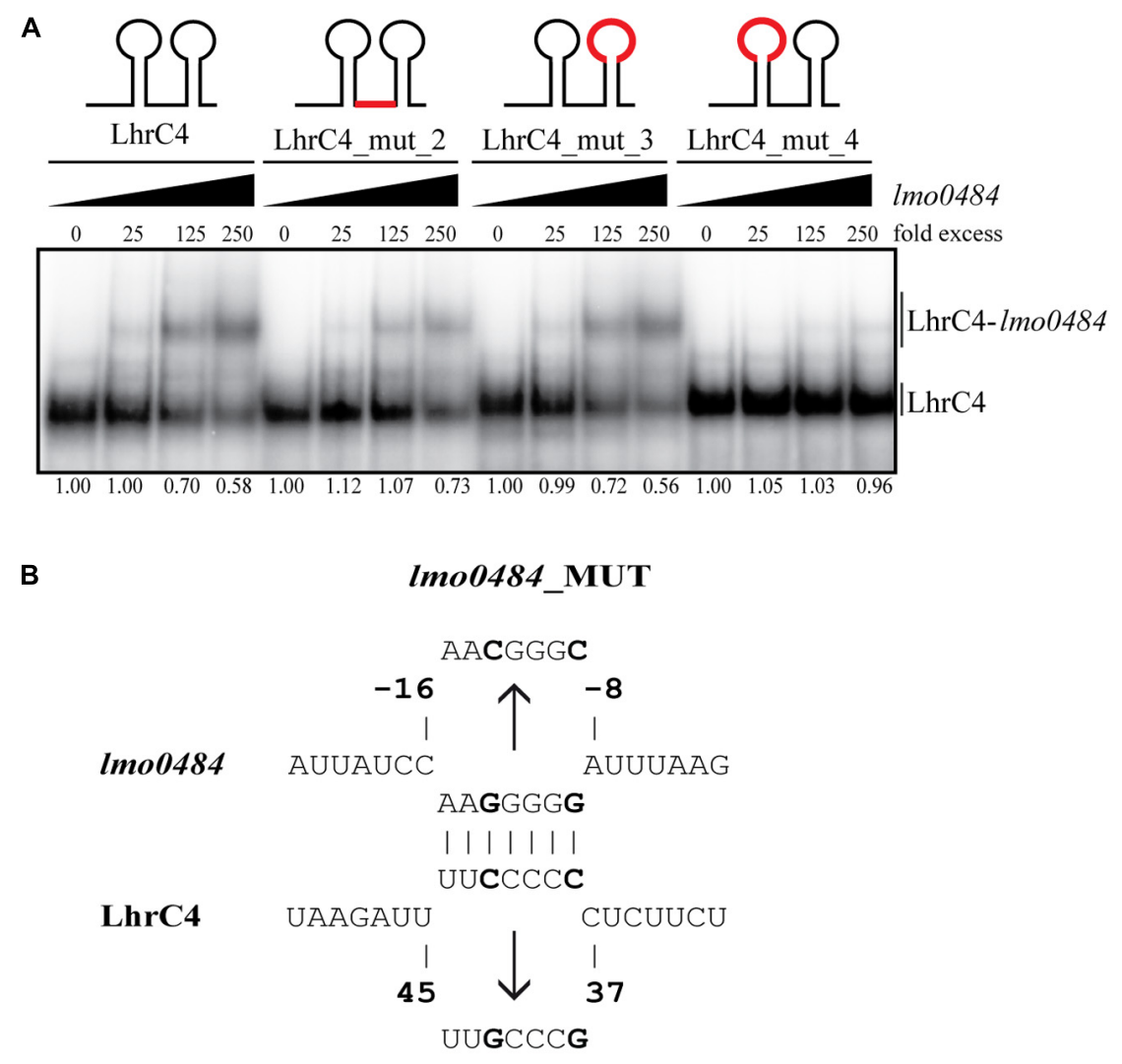

\section{LhrC4_loopA_MUT}

C

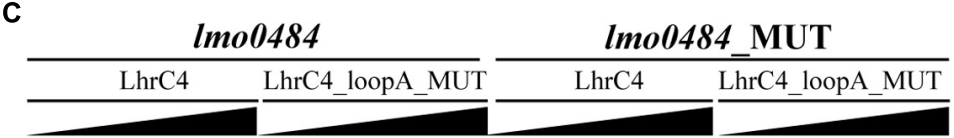

$\begin{array}{llllllllllllllllllll}0 & 4 & 20 & 100 & 500 & 0 & 4 & 20 & 100 & 500 & 0 & 4 & 20 & 100 & 500 & 0 & 4 & 20 & 100 & 500 \text { fold excess }\end{array}$

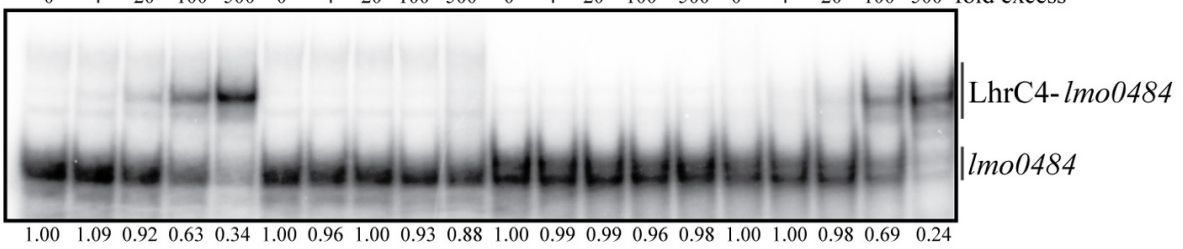

FIGURE 5 | Electrophoretic mobility shift assays (EMSAs) of the interaction between LhrC4 and Imo0484 mRNA. (A) LhrC4 mutant screening of loop A, single-stranded stretch, and the terminator loop. Labeled LhrC4 and the mutant derivatives were tested for their ability to interact with unlabeled Imo0484 RNA. The mutated regions are shown in red in the sRNA sketches. LhrC4: wild-type LhrC4; LhrC4_mut_2: mutation in single-stranded stretch; LhrC4_mut_3: mutation in terminator loop; LhrC4_mut_4: mutation in loop A. The fraction of unbound LhrC4 is shown below each lane. (B) Predicted basepairing between the SD region of Imo0484 mRNA and loop A of LhrC4. The mutated nucleotides are shown in bold and the sequences of the minimal mutant variants Imo0484_MUT and LhrC4_loopA_MUT are indicated. (C) Labeled Imo0484 RNA and Imo0484_MUT were each incubated with increasing concentrations of unlabeled LhrC4 or the mutant variant LhrC4_loopA_MUT. The fraction of unbound Imo0484 RNA is shown below each lane.

\section{Imo0484 Is Strongly Repressed in Response to Hemin Exposure and Is Dispensable for Growth Under Hemin Stress Conditions}

Based on the results obtained so far, we reasoned that the LhrCs could down-regulate the expression of $1 m o 0484$ in response to hemin stress. To test this hypothesis, wild-type and $\Delta l h r C 1-5$ cells containing the $\mathrm{pC}$-lmo0484-lacZ plasmid were exposed to hemin stress for 1 and $2 \mathrm{~h}$, and non-stressed cultures were used as controls. Upon the addition of hemin, the $\beta$-galactosidase activity in the wild-type and $\Delta l h r C 1-5$ cells remained at relatively low levels (Figure 8A and Supplementary Figure S2D), which is in stark contrast to the increase in Imo0484-lacZ expression 
A

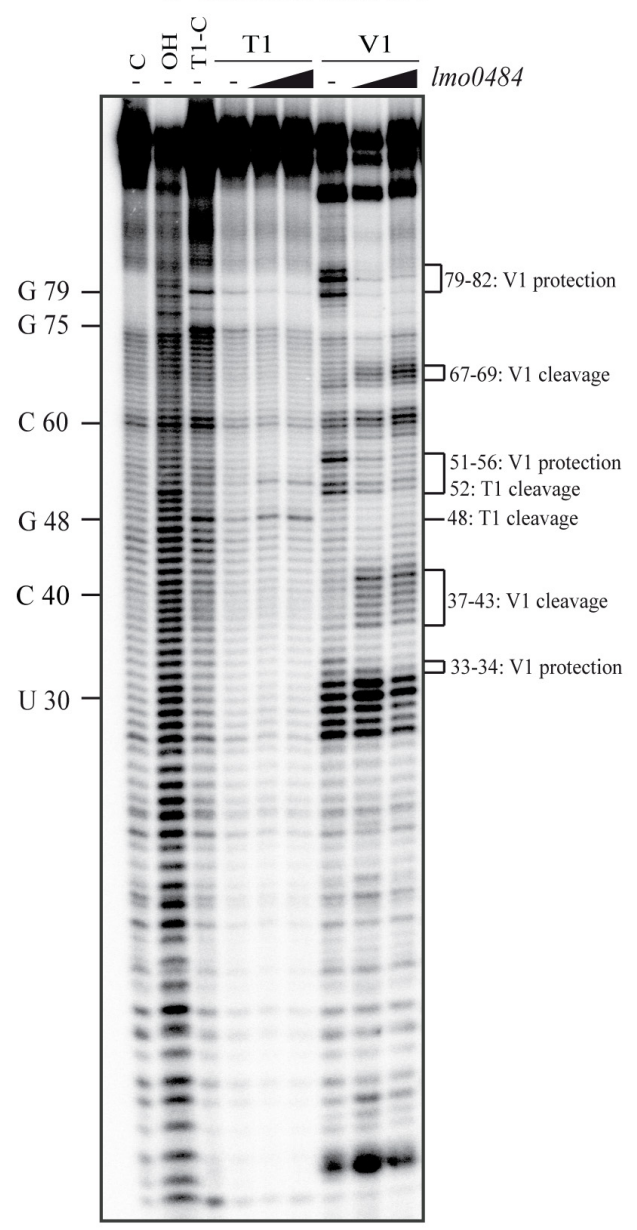

C

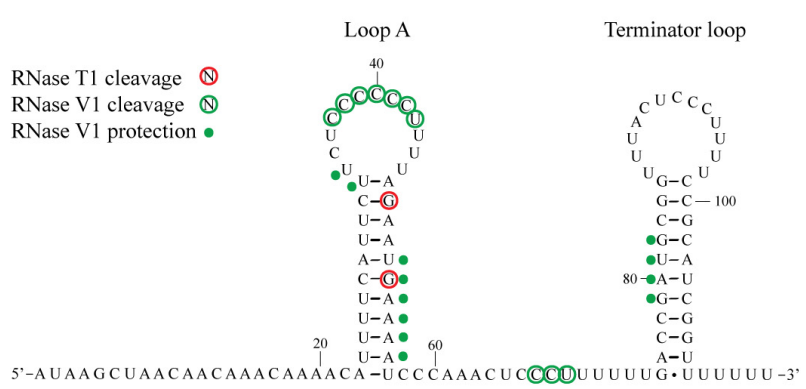

LhrC4
B

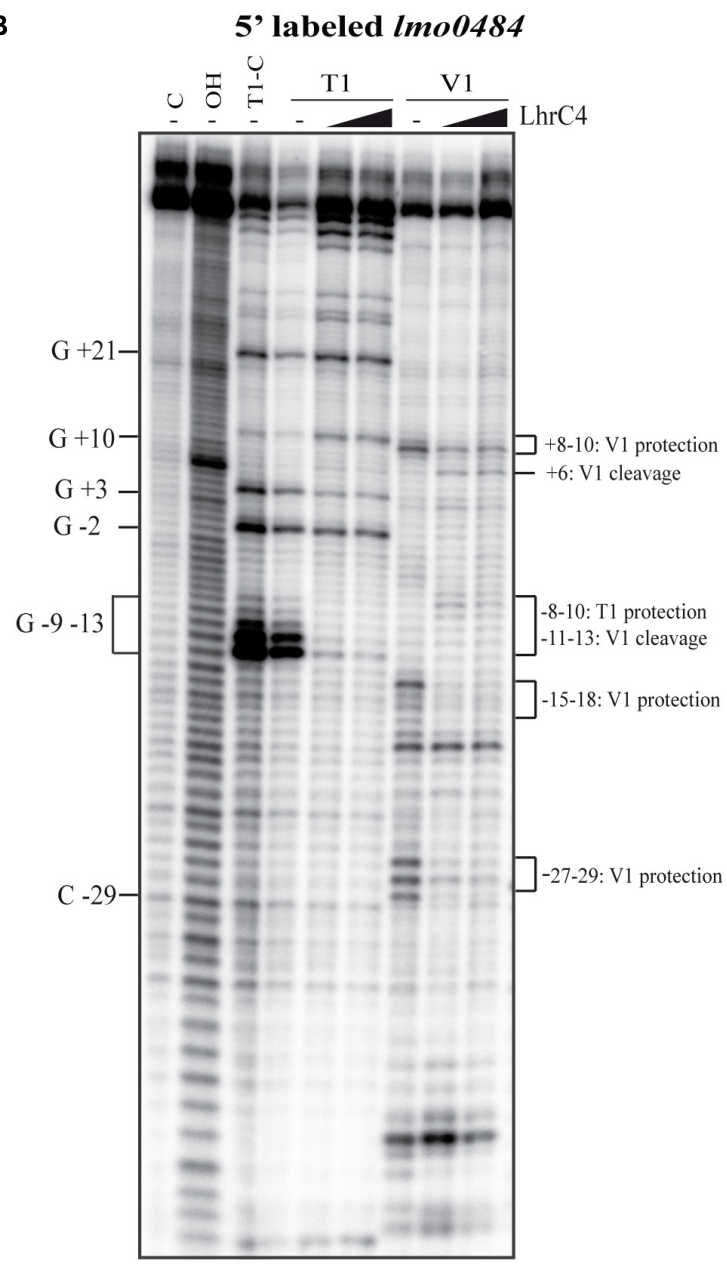

D

RNase V1 cleavage $\mathbb{Q}$ RNase T1 protection RNase V1 protection -

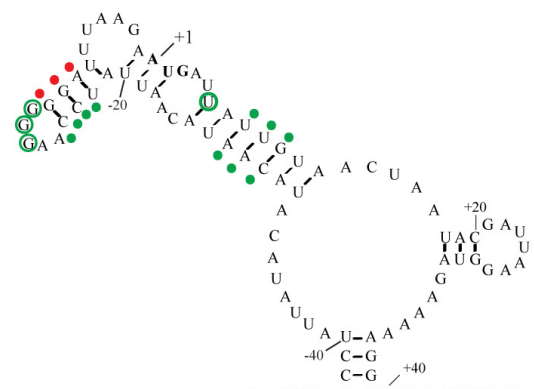

Imo0484 mRNA truncated

FIGURE 6 | Structure probing of LhrC4 and Imo0484 mRNA interaction. (A) 5'-end labeled LhrC4 was treated with RNase T1 or RNase V1, either in the absence (-) or in the presence of 25- or 250-fold excess of unlabeled Imo0484 RNA. As a control, untreated LhrC4 was separated in the first lane (C), an alkaline ladder (OH) is shown in the second lane and an RNase T1 ladder (T1-C) was separated in the third lane. For an overview, selected nucleotides are labeled on the left side. Nucleotides showing structural changes upon Imo0484 binding are marked on the right side of the gel. (B) Partial digestion of $5^{\prime}$-end labeled Imo0484 RNA with RNases T1 and V1. Untreated Imo0484 RNA (C), an alkaline ladder (OH) and an RNase T1 ladder (T1-C) are shown in the first three lanes. Some of the cleaved G residues are labeled along the left side of the gel. The Imo0484 nucleotides, that were protected or cleaved in the presence of LhrC4, are indicated on the right side of the gel. (C) Secondary structure of LhrC4 illustrating the cleavage pattern upon binding to Imo0484 mRNA. Residues cleaved by RNase T1 (red) or RNase V1 (green) are encircled. Residues of LhrC4, that appeared to be protected by Imo0484 RNA, are indicated by green dots. The nucleotides of LhrC4 are numbered relative to the $5^{\prime}$-end of the sRNA. (D) Secondary structure of the truncated version of Imo0484 mRNA showing an altered cleavage pattern upon the addition of LhrC4. Residues cleaved by RNase V1 (green) are encircled. Residues of Imo0484 RNA that were protected in the presence of LhrC4 are indicated by red dots ( 1 1 protection) or green dots (V1 protection). The start codon is indicated in bold. The nucleotides of Imo0484 are numbered relative to the translation start site (+ 1 ). 


\section{A pC-lmo0484-lacZ - Cefuroxime}

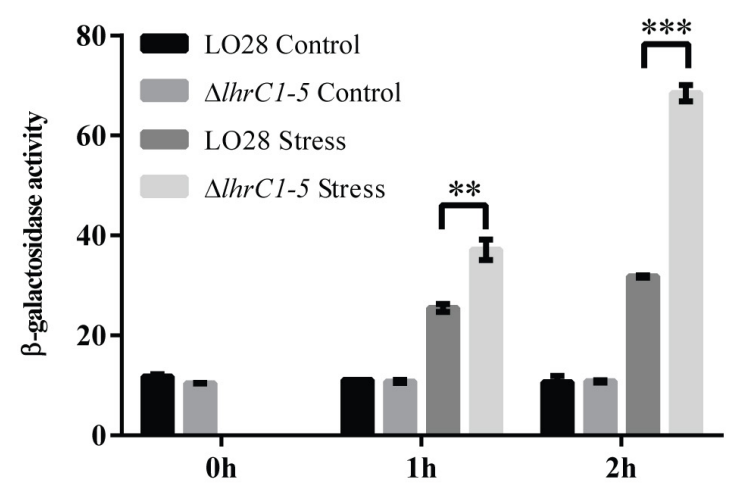

B

$\operatorname{lmo0484}$

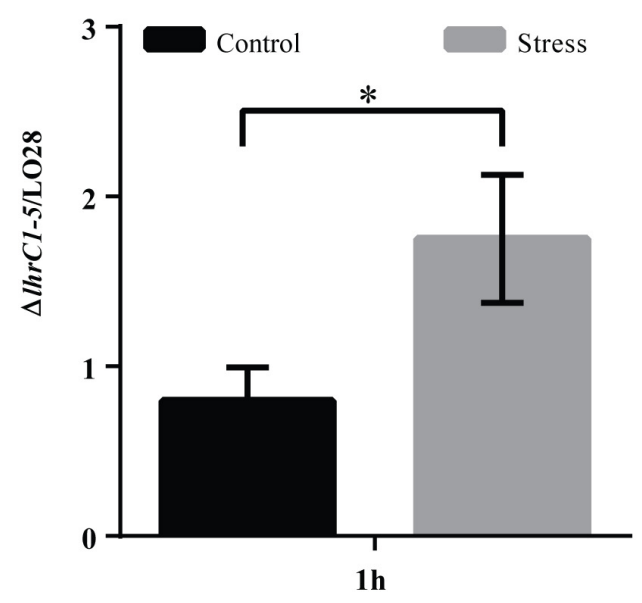

FIGURE 7 | Assessing the effect of LhrC1-5 on the novel target gene Imo0484 upon cefuroxime exposure. (A) $\beta$-galactosidase assay of LO28 wild-type and $\Delta / h r C 1-5$ strains carrying a translational reporter gene fusion of Imo0484 to lac Z in the vector pCK-lac. $\beta$-galactosidase activities of LO28 wild-type and mutant cells were measured at the indicated time points under non-stress conditions (Control) and after exposure to $9 \mu \mathrm{M}$ cefuroxime (Stress). The results are the average of three biological replicates, each carried out in technical duplicates. After 1 and $2 \mathrm{~h}$ of stress, a significant difference between the mutant and wild-type cells was observed $(* * p<0.001$;

${ }^{* * *} p<0.0001$ ). (B) Quantification of Imo0484 mRNA in $\Delta / h r C 1-5$ relative to LO28 wild-type by RT-qPCR. The ratio of $\Delta / h r C 1-5 / L O 28$ was determined at the $1 \mathrm{~h}$ time point for both non-stressed (Control) and cefuroxime-exposed samples (Stress). The result shown is the average of three biological replicates. The asterisk indicates a significant increase of the ratio under stress conditions compared to the control with $p<0.05$.

observed upon cefuroxime exposure (Figure 7A). After 2 h of hemin stress, the lmo0484-lacZ expression was only slightly, but significantly higher in the $\Delta l h r C 1-5$ cells relative to the wild-type cells (Figure 8A). These results prompted us to investigate the effect of hemin stress on $\operatorname{lmo0484}$ at the RNA level. First, the expression of $1 m o 0484$ was investigated in wild-type and $\Delta$ lhrC1-5 cells subjected to increasing concentrations of hemin for $1 \mathrm{~h}$ (Figure 8B). The results of the northern blot experiment clearly demonstrated a strong repression of $1 m o 0484$ transcript levels in the wild-type strain already at the lowest concentration of hemin tested. For comparison, the lmo0484 mRNA level in the wild-type strain remained unaffected by $9 \mu \mathrm{M}$ cefuroxime (Cef). When comparing the wild-type and $\Delta / h r C 1-5$ cells after $1 \mathrm{~h}$ of hemin exposure, a regulatory effect of LhrC1-5 on 1 mo0484 was not evident, although LhrC1-5 was clearly expressed (Figure 8B).

To further explore the response of L. monocytogenes to excess hemin, a time course experiment was performed. Using northern blot analysis, the levels of LhrC1-5 and 1 mo0484 mRNA were determined in wild-type and $\Delta / h r C 1-5$ cells at various time points after hemin exposure (Figure $8 \mathrm{C}$ ). In the wild-type background, LhrC1-5 were clearly detected just 5 min after the addition of hemin, reaching a peak after $20 \mathrm{~min}$ of stress. A strong decrease in the transcript level of $1 m 00484$ was observed after $5 \mathrm{~min}$ of stress, demonstrating that L. monocytogenes responds very quickly to excess hemin by shutting down the expression of $1 m o 0484 \mathrm{mRNA}$. Once again, no major differences were observed when comparing the lmo0484 mRNA levels in the wild-type and $\Delta l h r C 1-5$ strains, suggesting that additional regulatory factors are involved in mediating the repression of lmo0484 (Figure 8C). Indeed, a Fur box is present upstream from lmo0484 (Supplementary Figure S4), suggesting that the iron-responsive regulator Fur could be a negative regulator of lmo0484 expression. To investigate this, the $l m o 0484$ levels were tested in a mutant strain lacking the Fur regulator (Figure 8D). The results clearly demonstrate that Fur is not responsible for down-regulating the expression of $1 \mathrm{mo} 0484$ in response to hemin stress, and LisR seems not to be required either. Curiously, lmo2185 and lmo2186 transcript levels in the wild-type strain were also strongly repressed by the lowest concentration of hemin tested, and similarly, Fur was not responsible for the down-regulation observed (Supplementary Figures S4, S5).

In Gram-positive bacteria, members of the IsdG family are known to be required for the utilization of heme as an iron source (Reniere et al., 2010; Sheldon and Heinrichs, 2015). In addition, IsdG in Bacillus anthracis also acts to protect the bacterium against heme-mediated toxicity (Skaar et al., 2006). To assess the role of $l m o 0484$ during growth of L. monocytogenes under hemin stress conditions, a mutant strain lacking lmo0484 was constructed. No significant difference was observed when comparing the growth of the $\Delta l m o 0484$ and wild-type strains under hemin stress (Figure 8E).

To summarize, the mRNA level of $l m o 0484$ is quickly diminished upon hemin exposure by an unknown mechanism. Likewise, the level of lmo2186-2185 mRNA is strongly reduced under hemin-rich conditions. It is possible that LhrC1-5 act to ensure an efficient shut-down of the translation of the few lmo0484 and lmo2186-2185 mRNAs remaining after the onset of hemin exposure. In accordance with the expression profile of Imo0484 upon hemin exposure, this IsdG-encoding gene does not contribute to growth under hemin stress conditions.

\section{DISCUSSION}

Bacteria are known to utilize heme as an iron source during infection, however, in heme-rich environments, such as in 
A

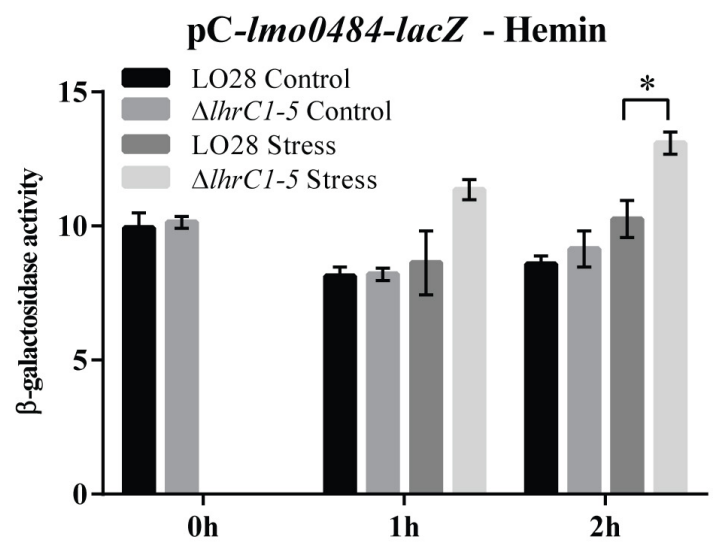

B

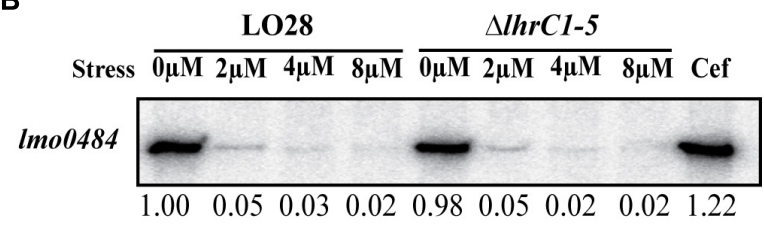

LhrC

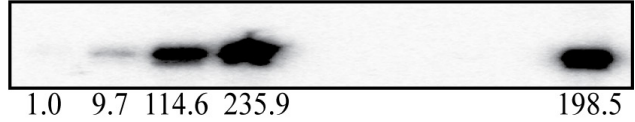

$5 S$

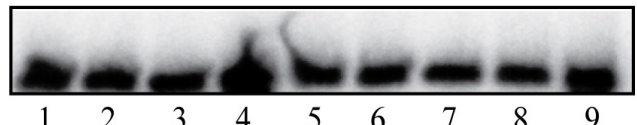

C

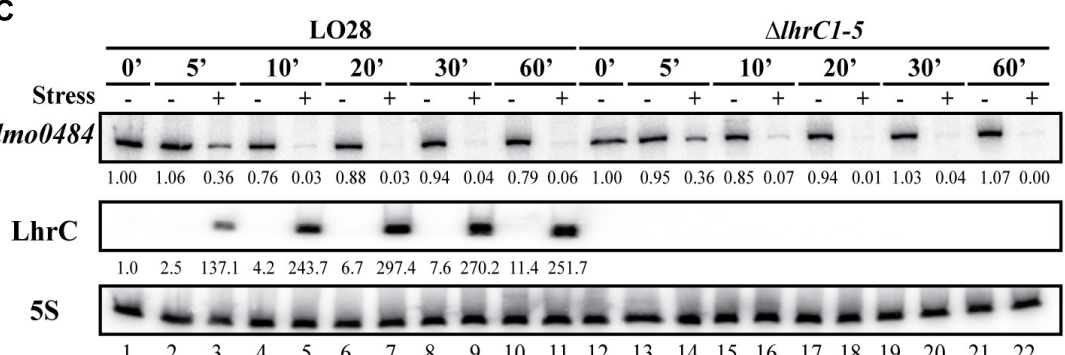

D

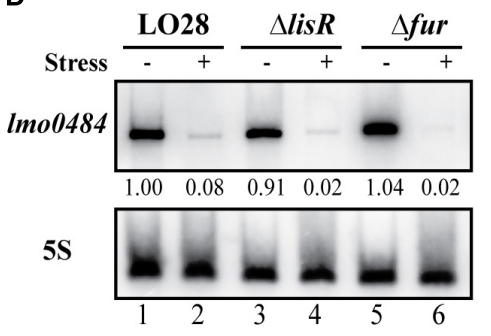

E

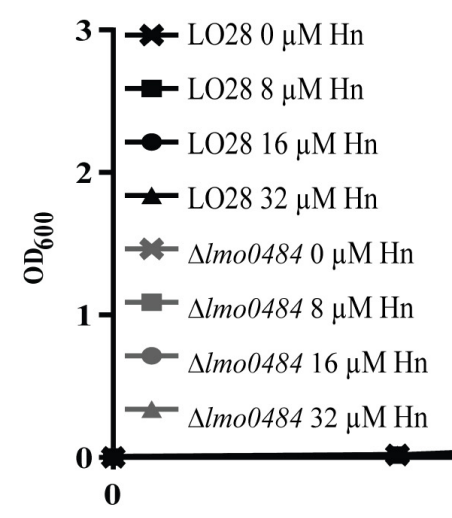

LO28 vs. $\Delta \operatorname{lmo0484}$

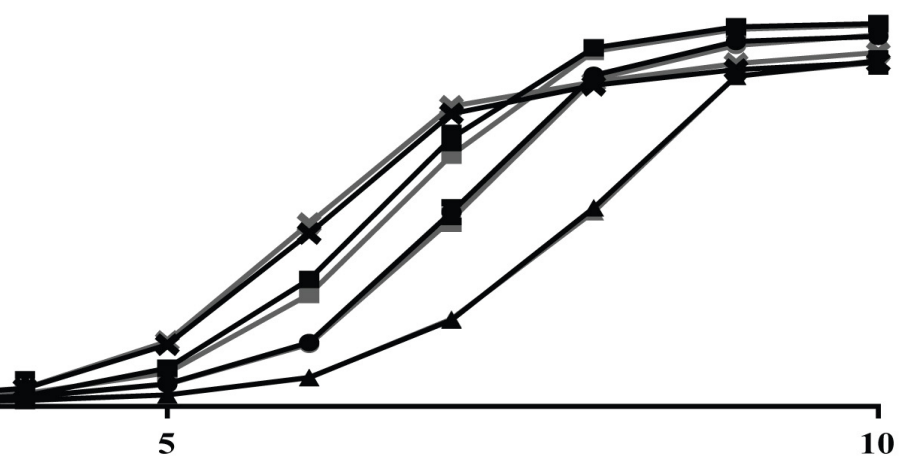

Time (h)

FIGURE 8 | Assessing the effect of LhrC1-5 on the novel target gene Imo0484 under hemin stress. (A) $\beta$-galactosidase assay of LO28 wild-type and $\Delta /$ /hrC1-5 strains carrying a translational reporter gene fusion of Imo0484 to lac $Z$ in the vector pCK-lac. $\beta$-galactosidase activities of wild-type and mutant cells were measured at the indicated time points under non-stress conditions (Control) and after exposure to $8 \mu \mathrm{M}$ hemin (Stress). The results are the average of three biological replicates, each carried out in technical duplicates. After $2 \mathrm{~h}$ of stress, a significant difference between the mutant and wild-type cells was observed ( $p<0.005)$. (B) Northern blot analysis of Imo0484 mRNA after exposure to various concentrations of hemin. Samples were taken from LO28 wild-type and $\Delta$ /hrC1-5 cultures exposed to $1 \mathrm{~h}$ of $0,2,4$, or $8 \mu \mathrm{M}$ hemin stress. A sample from LO28 wild-type culture exposed to $9 \mu \mathrm{M}$ cefuroxime was used as a control. Northern blots were probed for Imo0484 mRNA, LhrC1-5 and 5S rRNA (loading control). Relative levels of Imo0484 mRNA and LhrC1-5 (normalized to 5S) are shown below each lane. (C) LhrC induction profile and time-dependent regulation of Imo0484 mRNA in response to hemin stress. At time = 0, corresponding to OD $600=0.35$, LO28 wild-type and $\Delta / h r C 1-5$ cells were split in two, and one half of the culture was treated with $8 \mu M$ hemin (+), whereas the other half was left untreated (-). Samples were harvested at several time points $(0,5,10,20,30$, and 60 min relative to the addition of hemin) and total RNA was prepared for northern blot analysis. The blot was probed for Imo0484 mRNA, LhrC1-5 and 5S rRNA (loading control). Relative levels of Imo0484 mRNA and LhrC1-5 were normalized to 5S and are shown below each lane. (D) Testing the role of LisR and Fur in the heme-dependent repression of Imo0484. Samples were taken from LO28 wild-type, $\Delta$ lis $R$ and $\Delta$ fur cultures exposed for $1 \mathrm{~h}$ to $8 \mu \mathrm{M}$ hemin stress (+) as well as from non-stressed cultures (-). Northern blots were probed for Imo0484 mRNA and $5 \mathrm{~S}$ rRNA (loading control). Relative levels of Imo0484 mRNA normalized to $5 S$ are shown below each lane. (E) Stress tolerance assay of the $\Delta / m o 0484$ strain compared to LO28 wild-type. Wild-type and $\Delta / \mathrm{mo} 0484$ strains were grown in $\mathrm{BHI}$ containing $0,8,16$, or $32 \mu \mathrm{M}$ hemin. Bacterial growth was monitored until all cultures reached stationary phase. The average of three biological replicates is shown. 
the bloodstream and blood-rich organs, successful pathogens must defend themselves against the harmful effects of heme toxicity (Choby and Skaar, 2016; Joubert et al., 2017). Indeed, during severe hemolysis, free heme may reach concentrations up to $20 \mu \mathrm{M}$ (Arruda et al., 2004). Information on how L. monocytogenes maintains heme homeostasis is relatively limited and biased toward studies on the acquisition of hemoglobin and heme under iron-limited conditions (Klebba et al., 2012; Lechowicz and Krawczyk-Balska, 2015). In this study, we focused on how L. monocytogenes senses and responds to excess heme. We report that heme generates a signal that stimulates LisR-mediated activation of members of the LhrC family of sRNAs. Furthermore, we show that LisR and LhrC1-5 contribute to the adaptation of L. monocytogenes to growth under conditions of excess heme. LisRK has previously been found to be important for infection in mice (Cotter et al., 1999; Kallipolitis and Ingmer, 2001), and, together with LhrC1-5, LisR contributes to the intracellular replication of L. monocytogenes in macrophage-like cells (Sievers et al., 2015). The expression of LhrC1-5 is known to be highly induced when L. monocytogenes is exposed to whole human blood (Toledo-Arana et al., 2009), which corresponds well with a role for the LisR-regulated sRNAs in the adaptation to heme-rich environments.

We have previously shown that LhrC1-5 are induced in a LisR-dependent manner under cell envelope stress conditions, such as exposure to the cell wall-acting antibiotic cefuroxime, and that LhrC1-5 down-regulate the expression of cell envelope-associated proteins with virulence functions (Sievers et al., 2014, 2015). In the present study, we show that LhrC1-5 also repress the expression of the three known target genes in response to heme toxicity, suggesting that a fine-tuning of the levels of the cell envelope-associated proteins TcsA, OppA, and LapB is beneficial for L. monocytogenes under heme-rich conditions. Notably, whole human blood contains multiple components participating in host immunity, and surface exposed proteins are readily recognized by the immune system (Sievers et al., 2015). Thus, in heme-rich conditions, such as the human blood, LhrC-mediated down-regulation of surface exposed proteins may be viewed as an attempt by L. monocytogenes to evade detection by the immune system. Interestingly, the present study suggests that genes encoding cell envelope-associated proteins with known functions in heme acquisition belong to the LhrC regulon as well. The LhrC4 sRNA bound readily to the SD regions of $l m o 2186$-lmo2185 mRNA, encoding the heme uptake proteins $\mathrm{Hbp} 1$ and $\mathrm{Hbp} 2$, respectively. Additionally, the $1 m 00484$ gene, encoding a heme oxygenase, was found to be a target for LhrC regulation. The LhrC sRNAs were shown to down-regulate the expression of $1 m o 0484$ in response to cell envelope stress and LhrC4 was found to bind specifically to the SD region of lmo0484 mRNA using one of its three CU-rich regions. These observations suggest that the sRNAs act to inhibit translation initiation of $1 m o 0484$ under LhrC-inducing conditions, such as upon exposure to cefuroxime or heme. Whereas LhrC-mediated down-regulation of $1 m 00484$ expression was clearly detected in response to cefuroxime stress, the regulatory effect of LhrC1-5 on $\operatorname{lmo0484}$ was less pronounced under heme stress due to a rapid decrease in $1 m o 0484 \mathrm{mRNA}$ levels following the addition of excess heme to the growth medium. Interestingly, a similar decrease was observed when testing the level of lmo2186-lmo2185 mRNA upon heme exposure, suggesting a common mechanism for down-regulation of genes involved in heme uptake and utilization in response to heme stress. Although potential Fur boxes were found in the promoter regions upstream from Imo2186-lmo2185 and Imo0484, the Fur regulator was not required for the heme-mediated repression. Thus, the mechanism underlying the repressive effect of excess heme on the heme uptake and utilization genes in L. monocytogenes remains elusive. Considering the potential damaging effects of heme, an instant shut-down of the heme uptake genes $\operatorname{lmo} 2186$ and lmo2185 in response to excess heme seems logical, whereas the repression of the heme oxygenase-encoding gene lmo0484 is more ambiguous. In other organisms, including the bacterial pathogen $B$. anthracis, heme oxygenases have been shown to contribute to the heme detoxification process (Skaar et al., 2006), but in L. monocytogenes, the heme oxygenase encoded by lmo0484 appears to be dispensable in heme-rich environments. Indeed, L. monocytogenes wild-type and $\Delta l m o 0484$ strains were found to grow equally well under heme stress conditions (Figure 8E). Whether the second heme oxygenase encoded by L. monocytogenes (Isd-LmHde) contributes to the heme detoxification process remains to be investigated.

Based on our findings, we propose the following model for LhrC-mediated control of the heme uptake and utilization genes lmo0484, lmo2186, and lmo2185 under cefuroxime stress and heme stress (see Figure 9): under non-stress conditions, these genes are clearly expressed (Figures 8B-D and Supplementary Figure S5), indicating that L. monocytogenes is using the heme already present in the BHI medium as a source of iron (Figure 9A); in response to cefuroxime stress, LhrC1-5 fine-tune the expression of $l m o 0484$, and most likely also $l m o 2186$ and lmo2185, suggesting that L. monocytogenes is employing sRNA-mediated control to maintain heme homeostasis under cell envelope stress conditions (Figure 9B); upon exposure to excess heme, the levels of $l m o 0484$ and lmo2186-lmo2185 mRNAs are strongly reduced by an unknown mechanism (e.g., heme-induced transcriptional repression and/or mRNA degradation) (Figure 9C). In this case, we speculate that the role of LhrC1-5 is to prevent translation of the remaining lmo0484 mRNA, and possibly also lmo2186-lmo2185 mRNA, following heme exposure. Notably, LhrC1-5 were induced more than 50 -fold after just $5 \mathrm{~min}$ of heme stress, showing that the LhrC sRNAs are available for basepairing to target mRNAs within a few minutes (Figure $\mathbf{8 C}$ ). Together, these regulatory mechanisms act to repress the expression of genes involved in heme uptake and utilization, which allows $L$. monocytogenes to adapt very quickly to heme-rich conditions. Collectively, this study shows that the detected outcome of LhrC-mediated control relies not only on the presence of the sRNAs, but also on the availability of the partner mRNAs, which may be subject to control by other regulatory mechanisms under specific stress conditions (Figure 9C).

The results obtained in this study clearly demonstrate a role for LisRK in the response to excess heme. In addition to LisRK, at least one more TCS is expected to contribute to the adaptation of L. monocytogenes to heme-rich conditions. In Gram-positive 


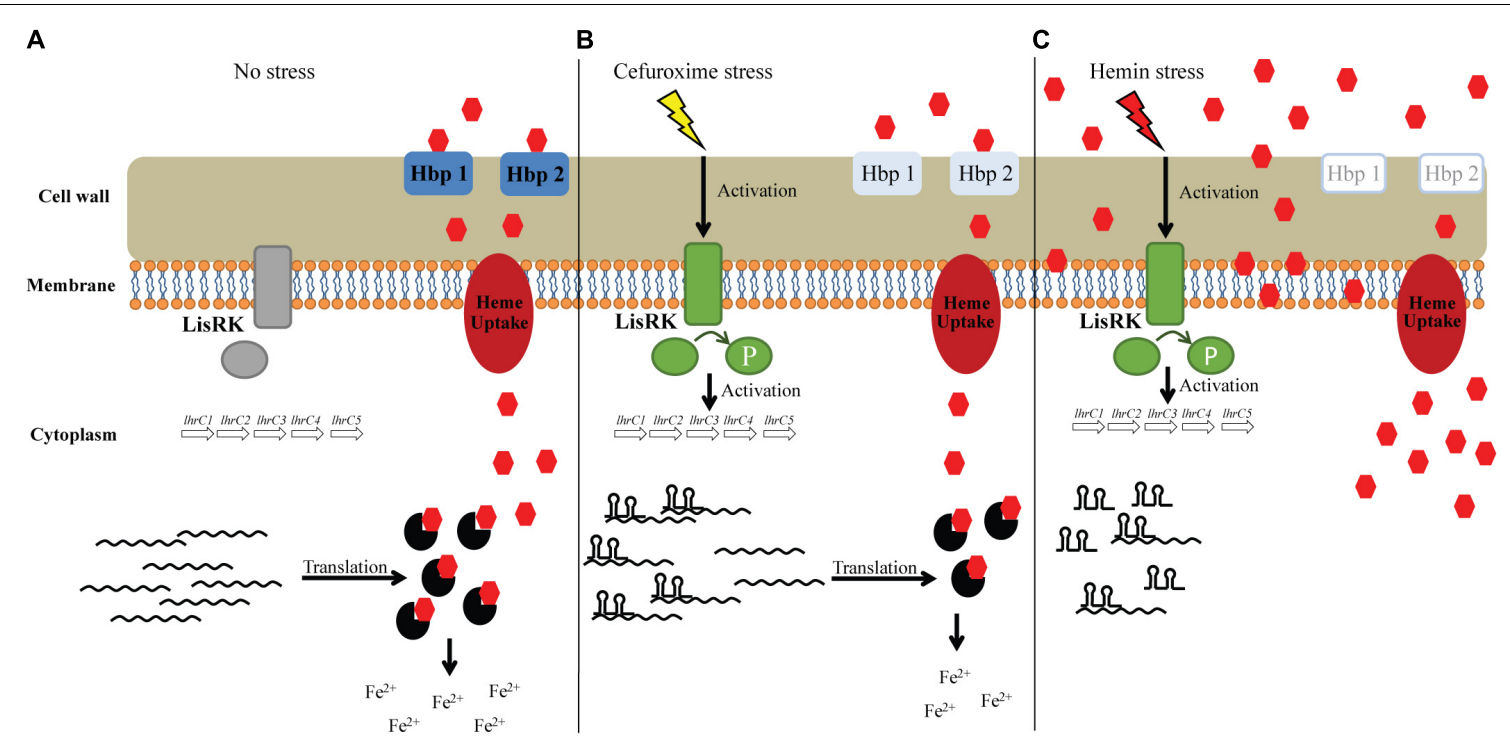

Heme Imo0484 LhrC sRNA $\Omega(\Omega)$ Lmo0484+Heme

FIGURE 9 | Proposed model of the regulatory effect of LhrC1-5 on heme uptake and utilization genes. (A) During growth in BHI medium, there is no activation of the TCS LisRK and subsequently LhrC1-5 are not induced. Under this condition, Imo0484, Imo2186, and Imo2185 are expressed and heme-uptake from the BHI medium is taking place according to the cells' needs. The Imo0484 mRNA is translated into a IsdG-like heme oxygenase, which will catalyze the degradation of heme to obtain free iron. (B) When the cells are subjected to cefuroxime stress, LisRK will be activated. LhrC1-5 are then induced and can bind Imo0484 mRNAs. Binding of LhrC sRNA to the SD region of Imo0484 mRNA leads to inhibition of translation. Under cefuroxime stress, LhrC1-5 most likely act to control the expression of the heme uptake genes Imo2186 and Imo2185 in a similar fashion (not shown). Thus, when exposed to cefuroxime, LhrC1-5 act to fine-tune the expression of heme uptake and utilization genes, suggesting a need for $L$. monocytogenes to slightly reduce the uptake of heme when the cell envelope is being stressed. (C) Under hemin stress, LhrC1-5 are strongly induced as well in a LisR-dependent manner. Furthermore, when the cell faces high concentrations of heme, the mRNA levels of Imo0484 are almost immediately diminished by an unknown mechanism. In this situation, LhrC1-5 will bind the remaining Imo0484 mRNAs in the cell and act as 'vacuum cleaners' to avoid translation of the residual Imo0484 mRNA. The heme uptake genes Imo2185 and Imo2186 are most likely controlled by heme and LhrC1-5 in a similar fashion (not shown). Consequently, the expression of heme acquisition genes is quickly reduced in response to heme stress to avoid further uptake of heme and liberation of iron.

pathogens, the TCS HssR has been shown to play a major role in the response to heme stress (Choby and Skaar, 2016). In $S$. aureus and B. anthracis, HssRS controls the expression of genes encoding a heme-regulated transporter, HrtAB, which protects the bacteria from heme toxicity by exporting heme (Torres et al., 2007; Stauff and Skaar, 2009). The HssRS system and $\mathrm{HrtAB}$ exporter are conserved in L. monocytogenes as well (Torres et al., 2007), however, their roles in dealing with heme toxicity remains to be clarified. Curiously, the HssRS system of $B$. anthracis was recently found to interact with another TCS, HitRS, which responds to compounds that affect the cell envelope integrity (Mike et al., 2014). HssRS and HitRS both act to stimulate $h r t A B$ expression in response to heme and cell envelope stress, respectively. Furthermore, the histidine kinase HssS was shown to cross-phosphorylate the response regulator HitR to activate the expression of the hitPQRS operon upon heme exposure (Mike et al., 2014). This operon encodes components of an unstudied $\mathrm{ABC}$ transporter and the HitRS system. The cross-regulation between HssRS and HitRS is thought to ensure a coordinated response to heme and cell envelope stress in B. anthracis, which may enable this pathogen to better adapt and survive during infection (Mike et al., 2014). We found that heme stimulates LisR-dependent activation of LhrC1-5 as efficiently as the cell-wall acting antibiotic cefuroxime, revealing a link between the response to heme toxicity and cell envelope stress in L. monocytogenes as well. Future studies should focus on clarifying the interconnections (if any) between LisRK and the putative heme-responsive HssRS system in L. monocytogenes. Most importantly, it should be investigated if LisRK cross-phosphorylates with HssRS, as shown for HitRS and HssRS in B. anthracis (Mike et al., 2014).

In addition to the LhrCs in L. monocytogenes, a hemeresponsive sRNA has also been described in Pseudomonas aeruginosa. The sRNA PrrH is encoded from the prrF locus in $P$. aeruginosa and overlaps with two iron-regulated sRNAs, PrrF1, and PrrF2 (Oglesby-Sherrouse and Vasil, 2010). In contrast to LhrC1-5, which are highly induced in response to excess heme, the PrrH sRNA is repressed by heme via an unknown mechanism. Furthermore, PrrH is repressed by iron, most likely via the Fur protein in $P$. aeruginosa. Although $\mathrm{PrrH}$ has been predicted to regulate genes involved in heme homeostasis and virulence through the unique sequence derived from the prrF1-prrF2 intergenic region (Oglesby-Sherrouse and Vasil, 2010; Reinhart et al., 2015), a recent study showed that PrrH is not required for acute murine lung infection (Reinhart et al., 2017). Notably, the region unique to $\mathrm{PrrH}$ is highly conserved across $P$. aeruginosa 
strains of clinical origin, suggesting that a role for $\operatorname{PrrH}$ could be found using alternative infection models (Reinhart et al., 2017). The finding of heme-regulated sRNAs in both L. monocytogenes and $P$. aeruginosa raises the possibility of a more widespread role for sRNA-mediated control in the response of bacterial pathogens to heme.

\section{AUTHOR CONTRIBUTIONS}

PdS, PM-G, DS, and BK: conceived and designed the experiments. PdS, PM-G, DS, J-HC, MB, and EL: performed the experiments. PdS, PM-G, DS, J-HC, MB, EL, and BK: analyzed the data. PdS and BK: wrote the paper. All authors read and approved the final manuscript.

\section{REFERENCES}

Arruda, M. A., Rossi, A. G., de Freitas, M. S., Barja-Fidalgo, C., and GracaSouza, A. V. (2004). Heme inhibits human neutrophil apoptosis: involvement of phosphoinositide 3-kinase, MAPK, and NF-kappaB. J. Immunol. 173, 2023-2030. doi: 10.4049/jimmunol.173.3.2023

Busch, A., Richter, A. S., and Backofen, R. (2008). IntaRNA: efficient prediction of bacterial sRNA targets incorporating target site accessibility and seed regions. Bioinformatics 24, 2849-2856. doi: 10.1093/bioinformatics/btn544

Cassat, J. E., and Skaar, E. P. (2013). Iron in infection and immunity. Cell Host Microbe 13, 509-519. doi: 10.1016/j.chom.2013.04.010

Chiabrando, D., Vinchi, F., Fiorito, V., Mercurio, S., and Tolosano, E. (2014). Heme in pathophysiology: a matter of scavenging, metabolism and trafficking across cell membranes. Front. Pharmacol. 5:61. doi: 10.3389/fphar.2014.00061

Chipperfield, J. R., and Ratledge, C. (2000). Salicylic acid is not a bacterial siderophore: a theoretical study. Biometals 13, 165-168. doi: 10.1023/A: 1009227206890

Choby, J. E., and Skaar, E. P. (2016). Heme synthesis and acquisition in bacterial pathogens. J. Mol. Biol. 428, 3408-3428. doi: 10.1016/j.jmb.2016.03.018

Christiansen, J. K., Larsen, M. H., Ingmer, H., Sogaard-Andersen, L., and Kallipolitis, B. H. (2004). The RNA-binding protein $\mathrm{Hfq}$ of Listeria monocytogenes: role in stress tolerance and virulence. J. Bacteriol. 186, 3355-3362. doi: 10.1128/JB.186.11.3355-3362.2004

Cotter, P. D., Emerson, N., Gahan, C. G., and Hill, C. (1999). Identification and disruption of lisRK, a genetic locus encoding a two-component signal transduction system involved in stress tolerance and virulence in Listeria monocytogenes. J. Bacteriol. 181, 6840-6843.

Duong, T., Park, K., Kim, T., Kang, S. W., Hahn, M. J., Hwang, H. Y., et al. (2014). Structural and functional characterization of an Isd-type haem-degradation enzyme from Listeria monocytogenes. Acta Crystallogr. D Biol. Crystallogr. 70(Pt 3), 615-626. doi: 10.1107/S1399004713030794

Foller, M., Shumilina, E., Lam, R., Mohamed, W., Kasinathan, R., Huber, S., et al. (2007). Induction of suicidal erythrocyte death by listeriolysin from Listeria monocytogenes. Cell Physiol. Biochem. 20, 1051-1060. doi: 10.1159/000110715

Geoffroy, C., Gaillard, J. L., Alouf, J. E., and Berche, P. (1987). Purification, characterization, and toxicity of the sulfhydryl-activated hemolysin listeriolysin O from Listeria monocytogenes. Infect. Immun. 55, 1641-1646.

Hammer, N. D., and Skaar, E. P. (2011). Molecular mechanisms of Staphylococcus aureus iron acquisition. Annu. Rev. Microbiol. 65, 129-147. doi: 10.1146/ annurev-micro-090110-102851

Hentze, M. W., Muckenthaler, M. U., and Andrews, N. C. (2004). Balancing acts: molecular control of mammalian iron metabolism. Cell 117, 285-297. doi: 10.1016/S0092-8674(04)00343-5

Huang, W., and Wilks, A. (2017). Extracellular heme uptake and the challenge of bacterial cell membranes. Annu. Rev. Biochem. 86, 799-823. doi: 10.1146/ annurev-biochem-060815-014214

Jin, B., Newton, S. M., Shao, Y., Jiang, X., Charbit, A., and Klebba, P. E. (2006). Iron acquisition systems for ferric hydroxamates, haemin and haemoglobin in

\section{FUNDING}

This work was supported by the European Union's Horizon 2020 Research and Innovation Program under the Marie Skłodowska-Curie grant agreement No. 641984, the Novo Nordisk Foundation, and a "La Caixa" Fellowship Grant for Post-graduate Studies, "La Caixa" Banking Foundation, Barcelona, Spain to PM-G.

\section{SUPPLEMENTARY MATERIAL}

The Supplementary Material for this article can be found online at: https://www.frontiersin.org/articles/10.3389/fmicb. 2018.00599/full\#supplementary-material

Listeria monocytogenes. Mol. Microbiol. 59, 1185-1198. doi: 10.1111/j.13652958.2005.05015.x

Joubert, L., Dagieu, J. B., Fernandez, A., Derre-Bobillot, A., Borezee-Durant, E., Fleurot, I., et al. (2017). Visualization of the role of host heme on the virulence of the heme auxotroph Streptococcus agalactiae. Sci. Rep. 7:40435. doi: 10.1038/ srep40435

Kallipolitis, B. H., and Ingmer, H. (2001). Listeria monocytogenes response regulators important for stress tolerance and pathogenesis. FEMS Microbiol. Lett. 204, 111-115. doi: 10.1111/j.1574-6968.2001.tb10872.x

Kallipolitis, B. H., Ingmer, H., Gahan, C. G., Hill, C., and Sogaard-Andersen, L. (2003). CesRK, a two-component signal transduction system in Listeria monocytogenes, responds to the presence of cell wall-acting antibiotics and affects beta-lactam resistance. Antimicrob. Agents Chemother. 47, 3421-3429. doi: 10.1128/AAC.47.11.3421-3429.2003

Klebba, P. E., Charbit, A., Xiao, Q., Jiang, X., and Newton, S. M. (2012). Mechanisms of iron and haem transport by Listeria monocytogenes. Mol. Membr. Biol. 29, 69-86. doi: 10.3109/09687688.2012.694485

Kochan, I. (1973). The role of iron in bacterial infections, with special consideration of host-tubercle bacillus interaction. Curr. Top. Microbiol. Immunol. 60, 1-30. doi: 10.1007/978-3-642-65502-9_1

Lechowicz, J., and Krawczyk-Balska, A. (2015). An update on the transport and metabolism of iron in Listeria monocytogenes: the role of proteins involved in pathogenicity. Biometals 28, 587-603. doi: 10.1007/s10534-015-9849-5

Ledala, N., Sengupta, M., Muthaiyan, A., Wilkinson, B. J., and Jayaswal, R. K. (2010). Transcriptomic response of Listeria monocytogenes to iron limitation and Fur mutation. Appl. Environ. Microbiol. 76, 406-416. doi: 10.1128/AEM. 01389-09

Lungu, B., Ricke, S. C., and Johnson, M. G. (2009). Growth, survival, proliferation and pathogenesis of Listeria monocytogenes under low oxygen or anaerobic conditions: a review. Anaerobe 15, 7-17. doi: 10.1016/j.anaerobe.2008.08.001

Malmirchegini, G. R., Sjodt, M., Shnitkind, S., Sawaya, M. R., Rosinski, J., Newton, S. M., et al. (2014). Novel mechanism of hemin capture by Hbp2, the hemoglobin-binding hemophore from Listeria monocytogenes. J. Biol. Chem. 289, 34886-34899. doi: 10.1074/jbc.M114.583013

Mann, M., Wright, P. R., and Backofen, R. (2017). IntaRNA 2.0: enhanced and customizable prediction of RNA-RNA interactions. Nucleic Acids Res. 45(W1), W435-W439. doi: 10.1093/nar/gkx279

McLaughlin, H. P., Hill, C., and Gahan, C. G. (2011). The impact of iron on Listeria monocytogenes; inside and outside the host. Curr. Opin. Biotechnol. 22, 194-199. doi: 10.1016/j.copbio.2010.10.005

McLaughlin, H. P., Xiao, Q., Rea, R. B., Pi, H., Casey, P. G., Darby, T., et al. (2012). A putative P-type ATPase required for virulence and resistance to haem toxicity in Listeria monocytogenes. PLoS One 7:e30928. doi: 10.1371/journal. pone.0030928

Mike, L. A., Choby, J. E., Brinkman, P. R., Olive, L. Q., Dutter, B. F., Ivan, S. J., et al. (2014). Two-component system cross-regulation integrates Bacillus anthracis response to heme and cell envelope stress. PLoS Pathog. 10:e1004044. doi: 10.1371/journal.ppat.1004044 
Mollerup, M. S., Ross, J. A., Helfer, A. C., Meistrup, K., Romby, P., and Kallipolitis, B. H. (2016). Two novel members of the LhrC family of small RNAs in Listeria monocytogenes with overlapping regulatory functions but distinctive expression profiles. RNA Biol. 13, 895-915. doi: 10.1080/15476286.2016.120 8332

Newton, S. M., Klebba, P. E., Raynaud, C., Shao, Y., Jiang, X., Dubail, I., et al. (2005). The svpA-srtB locus of Listeria monocytogenes: fur-mediated iron regulation and effect on virulence. Mol. Microbiol. 55, 927-940. doi: 10.1111/ j.1365-2958.2004.04436.x

Nielsen, J. S., Lei, L. K., Ebersbach, T., Olsen, A. S., Klitgaard, J. K., ValentinHansen, P., et al. (2010). Defining a role for Hfq in Gram-positive bacteria: evidence for Hfq-dependent antisense regulation in Listeria monocytogenes. Nucleic Acids Res. 38, 907-919. doi: 10.1093/nar/gkp1081

Oglesby-Sherrouse, A. G., and Vasil, M. L. (2010). Characterization of a hemeregulated non-coding RNA encoded by the prrF locus of Pseudomonas aeruginosa. PLoS One 5:e9930. doi: 10.1371/journal.pone.0009930

Parrisius, J., Bhakdi, S., Roth, M., Tranum-Jensen, J., Goebel, W., and Seeliger, H. P. (1986). Production of listeriolysin by beta-hemolytic strains of Listeria monocytogenes. Infect. Immun. 51, 314-319.

Pfaffl, M. W. (2001). A new mathematical model for relative quantification in real-time RT-PCR. Nucleic Acids Res. 29, e45. doi: 10.1093/nar/29.9.e45

Pfaffl, M. W., Horgan, G. W., and Dempfle, L. (2002). Relative expression software tool (REST) for group-wise comparison and statistical analysis of relative expression results in real-time PCR. Nucleic Acids Res. 30:e36. doi: 10.1093/nar/ 30.9.e36

Poyart, C., and Trieu-Cuot, P. (1997). A broad-host-range mobilizable shuttle vector for the construction of transcriptional fusions to beta-galactosidase in gram-positive bacteria. FEMS Microbiol. Lett. 156, 193-198. doi: 10.1016/ S0378-1097(97)00423-0

Reinhart, A. A., Nguyen, A. T., Brewer, L. K., Bevere, J., Jones, J. W., Kane, M. A., et al. (2017). The Pseudomonas aeruginosa PrrF small RNAs regulate iron homeostasis during acute murine lung infection. Infect. Immun. 85:e00764-16. doi: 10.1128/IAI.00764-16

Reinhart, A. A., Powell, D. A., Nguyen, A. T., O’Neill, M., Djapgne, L., Wilks, A., et al. (2015). The prrF-encoded small regulatory RNAs are required for iron homeostasis and virulence of Pseudomonas aeruginosa. Infect. Immun. 83, 863-875. doi: 10.1128/IAI.02707-14

Reniere, M. L., Torres, V. J., and Skaar, E. P. (2007). Intracellular metalloporphyrin metabolism in Staphylococcus aureus. Biometals 20, 333-345. doi: 10.1007/ s10534-006-9032-0

Reniere, M. L., Ukpabi, G. N., Harry, S. R., Stec, D. F., Krull, R., Wright, D. W., et al. (2010). The IsdG-family of haem oxygenases degrades haem to a novel chromophore. Mol. Microbiol. 75, 1529-1538. doi: 10.1111/j.1365-2958.2010. 07076.x

Schaferkordt, S., and Chakraborty, T. (1995). Vector plasmid for insertional mutagenesis and directional cloning in Listeria spp. Biotechniques 720-722, 724-725.

Sheehan, B., Klarsfeld, A., Msadek, T., and Cossart, P. (1995). Differential activation of virulence gene expression by PrfA, the Listeria monocytogenes virulence regulator. J. Bacteriol. 177, 6469-6476. doi: 10.1128/jb.177.22.6469-6476. 1995

Sheldon, J. R., and Heinrichs, D. E. (2015). Recent developments in understanding the iron acquisition strategies of gram positive pathogens. FEMS Microbiol. Rev. 39, 592-630. doi: 10.1093/femsre/fuv009

Sievers, S., Lund, A., Menendez-Gil, P., Nielsen, A., Storm Mollerup, M., Lambert Nielsen, S., et al. (2015). The multicopy sRNA LhrC controls expression of the oligopeptide-binding protein OppA in Listeria monocytogenes. RNA Biol. 12, 985-997. doi: 10.1080/15476286.2015.1071011

Sievers, S., Sternkopf Lillebaek, E. M., Jacobsen, K., Lund, A., Mollerup, M. S., Nielsen, P. K., et al. (2014). A multicopy sRNA of Listeria monocytogenes regulates expression of the virulence adhesin LapB. Nucleic Acids Res. 42, 9383-9398. doi: 10.1093/nar/gku630
Skaar, E. P., Gaspar, A. H., and Schneewind, O. (2006). Bacillus anthracis IsdG, a heme-degrading monooxygenase. J. Bacteriol. 188, 1071-1080. doi: 10.1128/JB. 188.3.1071-1080.2006

Skaar, E. P., and Schneewind, O. (2004). Iron-regulated surface determinants (Isd) of Staphylococcus aureus: stealing iron from heme. Microbes Infect. 6, 390-397. doi: 10.1016/j.micinf.2003.12.008

Stauff, D. L., and Skaar, E. P. (2009). Bacillus anthracis HssRS signalling to HrtAB regulates haem resistance during infection. Mol. Microbiol. 72, 763-778. doi: 10.1111/j.1365-2958.2009.06684.x

Stojiljkovic, I., and Perkins-Balding, D. (2002). Processing of heme and hemecontaining proteins by bacteria. DNA Cell Biol. 21, 281-295. doi: 10.1089/ 104454902753759708

Thorsing, M., Dos Santos, P. T., and Kallipolitis, B. H. (2017). Small RNAs in major foodborne pathogens: from novel regulatory activities to future applications. Curr. Opin. Biotechnol. 49, 120-128. doi: 10.1016/j.copbio.2017.08.006

Toledo-Arana, A., Dussurget, O., Nikitas, G., Sesto, N., Guet-Revillet, H., Balestrino, D., et al. (2009). The Listeria transcriptional landscape from saprophytism to virulence. Nature 459, 950-956. doi: 10.1038/nature08080

Torres, V. J., Stauff, D. L., Pishchany, G., Bezbradica, J. S., Gordy, L. E., Iturregui, J., et al. (2007). A Staphylococcus aureus regulatory system that responds to host heme and modulates virulence. Cell Host Microbe 1, 109-119. doi: 10.1016/j. chom.2007.03.001

Travaglini-Allocatelli, C. (2013). Protein machineries involved in the attachment of heme to cytochrome c: protein structures and molecular mechanisms. Scientifica 2013, 505714. doi: 10.1155/2013/505714

Vazquez-Boland, J. A., Kocks, C., Dramsi, S., Ohayon, H., Geoffroy, C., Mengaud, J., et al. (1992). Nucleotide sequence of the lecithinase operon of Listeria monocytogenes and possible role of lecithinase in cell-to-cell spread. Infect. Immun. 60, 219-230.

Vazquez-Boland, J. A., Kuhn, M., Berche, P., Chakraborty, T., DominguezBernal, G., Goebel, W., et al. (2001). Listeria pathogenesis and molecular virulence determinants. Clin. Microbiol. Rev. 14, 584-640. doi: 10.1128/CMR. 14.3.584-640.2001

Wakeman, C. A., and Skaar, E. P. (2012). Metalloregulation of Gram-positive pathogen physiology. Curr. Opin. Microbiol. 15, 169-174. doi: 10.1016/j.mib. 2011.11.008

Wright, P. R., Georg, J., Mann, M., Sorescu, D. A., Richter, A. S., Lott, S., et al. (2014). CopraRNA and IntaRNA: predicting small RNA targets, networks and interaction domains. Nucleic Acids Res. 42, W119-W123. doi: 10.1093/nar/ gku359

Wu, R., Skaar, E. P., Zhang, R., Joachimiak, G., Gornicki, P., Schneewind, O., et al. (2005). Staphylococcus aureus IsdG and IsdI, heme-degrading enzymes with structural similarity to monooxygenases. J. Biol. Chem. 280, 2840-2846. doi: 10.1074/jbc.M409526200

Xiao, Q., Jiang, X., Moore, K. J., Shao, Y., Pi, H., Dubail, I., et al. (2011). Sortase independent and dependent systems for acquisition of haem and haemoglobin in Listeria monocytogenes. Mol. Microbiol. 80, 1581-1597. doi: 10.1111/j.13652958.2011.07667.x

Zuker, M. (2003). Mfold web server for nucleic acid folding and hybridization prediction. Nucleic Acids Res. 31, 3406-3415. doi: 10.1093/nar/gkg595

Conflict of Interest Statement: The authors declare that the research was conducted in the absence of any commercial or financial relationships that could be construed as a potential conflict of interest.

Copyright ( 2018 dos Santos, Menendez-Gil, Sabharwal, Christensen, Brunhede, Lilleboek and Kallipolitis. This is an open-access article distributed under the terms of the Creative Commons Attribution License (CC BY). The use, distribution or reproduction in other forums is permitted, provided the original author(s) and the copyright owner are credited and that the original publication in this journal is cited, in accordance with accepted academic practice. No use, distribution or reproduction is permitted which does not comply with these terms. 